\title{
Metronomic anti-cancer therapy - an ongoing treatment option for advanced cancer patients
}

\author{
Mross $\mathrm{K}^{1+*}$ and Steinbild $\mathrm{S}^{\dagger 2}$ \\ ${ }^{*}$ Correspondence: mross@tumorbio.uni-freiburg.de \\ 'Department Medical Oncology in the Tumor Biology Center at the Albert-Ludwigs University Freiburg, Germany. \\ ${ }^{2}$ Medical office Division Oncology \& Hematology Neumarkt, Germany.
}

\begin{abstract}
The therapeutic concept of administering agents (cytotoxic/-static,non-cytotoxic and/or targeted drugs) continuously at lower doses - relative to MTDs in the case of cytostatic and cytotoxic drugs or continuously at tolerable doses as in the case of targeted drugs without drug-free breaks over extended periods - known as 'metronomic therapy' (MT), is increasingly being recognized as an experimental option for treating cancer. In comparison with MTD-defined chemotherapy (CHT) regimens, metronomic therapy has demonstrated reduced toxicity. More importantly, several phase II trials have shown that metronomic therapies showed anti-cancer activity in different cancer types with different drugs. The mechanistic basis of metronomic therapy using cytotoxic/static drugs is believed to be primarily anti-angiogenic, either by direct killing or inhibiting endothelial cells (ECs) in the tumor vasculature, killing bone-marrow-derived endothelial progenitor cells, stimulating the immune system, directly affecting tumor cells through a drug-driven effect as well as specificly inhibiting a target when targeting drugs were used in additional to metronomic therapy. The induction of senescence in cancer is another possible explanation for the principles behind 'metronomic therapy'.
\end{abstract}

Keywords: Metronomic therapy, anti-angiogenesis, cytotoxic drugs, cytostatic drugs, targeted drugs

\section{Introduction}

The concept of using anti-angiogenic approaches as an anti-cancer treatment strategy was suggested by Judah Folkman in 1971 [1]. Three decades later the first approval of a 'new' anti-angiogenic agent was attained [2], although the debate of how to use bevacizumab optimally in which patients is still ongoing. Several physicians argue that using anti-angiogenic agents such as bevacizumab continuously and not only as first line does not appear to be a realistic option due to the high costs involved. Many conventional cytotoxic drugs have significant antiangiogenic effects [3]. Conventional chemotherapy $\mathrm{CHT}$ is normally administered near the maximal tolerated dose (MTD), typically in 3-week cycles. When CHT is used in this way with a 3 or more weeks drug-free period, the host can recover from adverse effects and the vascular damage will rapidly be repaired whereas cancer cells have less chance of recovering. Nevertheless during the drug-free period, regrowth of parts of the cancer cells occurs, especially if the drug-free period is too long. It has been shown, that by shortening the drug-free period between $\mathrm{CHTs}$, the anti-angiogenic effects can be largely augmented [4] Unfortunately, the study of Browder et al., was carried out with rapidly proliferating tumors after subcutaneous transplantation in mice, but the use of slowgrowing, spontaneous tumors would be a better method to evaluate this topic. This as metronomic dubbed $\mathrm{CHT}$ can be optimized when combined with targeted agents [4]. A metronomic CHT is characterized by the following principles (see Table 1).

Table 1. Characteristics of metronomic chemotherapy

- frequent (dose-dense) CHT administration without any interruptions

- not using the maximal tolerated dose (MTD) include a biological optimized dose (BOD)

- no application of hematopoetic growth factors

- preference for oral drugs

- low incidence of treatment related side effects

- potential for delayed development of resistance

This approach can be further refined. In fact, the only available anti-neoplastic drugs which can be administered orally are limited to: cyclophosphamide, methotrexate, trofosfamide, capecitabine, S-1, UFT, navelbine, etoposid, temozolomide, dexamethasone, estramustin, chlorambucil,

(C) 2012 Mross et al; licensee Herbert Publications Ltd. This is an open access article distributed under the terms of Creative Commons Attribution License (http://creativecommons.org/licenses/by/3.0),This permits unrestricted use, distribution, and reproduction in any medium, provided the original work is properly cited. 


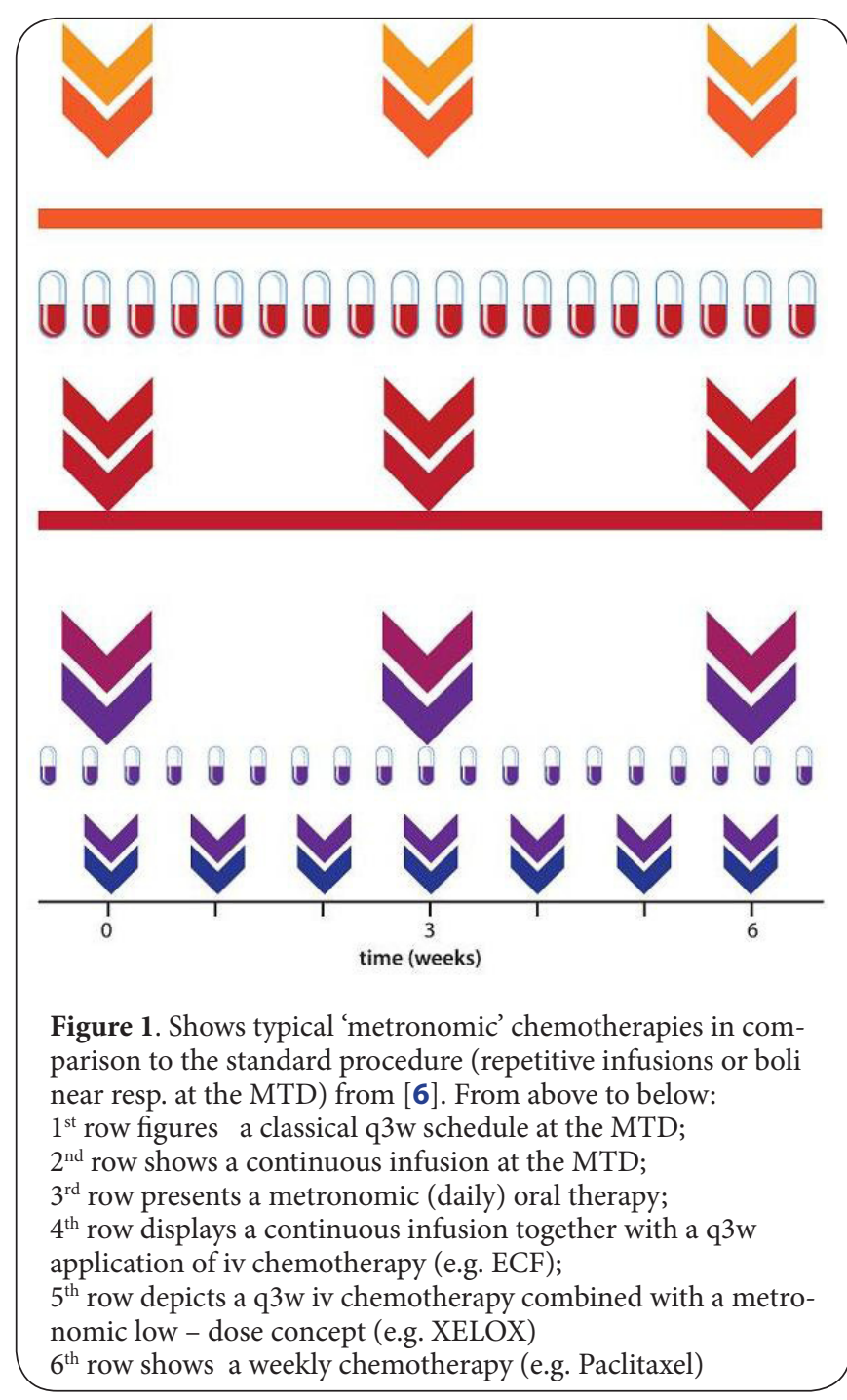

busulfan, 6-mercaptopurine, procarbacin, treosulfan, idarubicin, topotecan and hydroxycarbamid. The use of some of these drugs is limited because... not all are available in such low drug doses as it would be necessary for a daily (metronomic) treatment, because they were developed for oral CHT treatments, classically MTD-defined. Combinations with approved anti-angiogenic agents include bevacizumab, sunitinib, sorafenib, pazopanib, temsirolimus and other small molecules like imatinib, dasatinib, lapatinib, thalidomide and lenalidomide are possible.

Besides, there are some drugs, which were developed and approved not for anti-cancer treatment but for other indications which later showed anti-angiogenic potency among other effects: cyclooxygenase-2 inhibitors (COX-2) $[60,100,102]$, peroxisome proliferator-activated receptor- $\gamma$ agonists (PPAR $\gamma$ ) [82], metformin, nelfinarvir, nitoxoline, thalidomide and others $[106,107,112,113,116,119]$. All these drugs have, in addition to the effects for which they are approved, anti-angiogenic effects and can be given orally daily in a metronomic fashion.

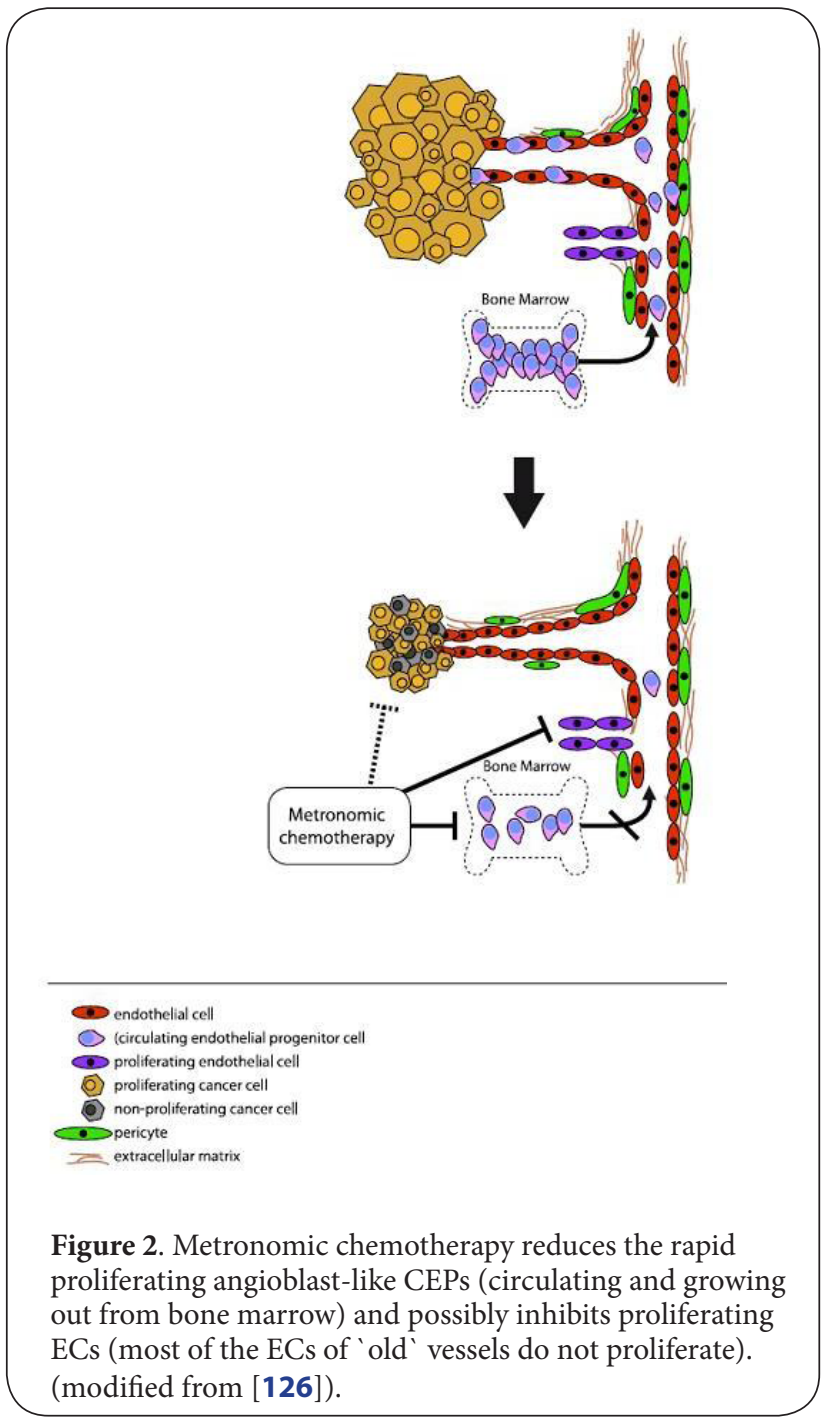

\section{Anti-angiogenic properties}

Growth of tumor vasculature depends on angiogenesis which is mediated by local sprouting of rapidly dividing endothelial cells (ECs) from pre-existing capillaries and vasculogenesis which requires circulating endothelial precursor cells (CEPs) (angioblast-like) from bone marrow [7]. Intratumoral vascular ECs proliferate rapidly in contrast to the ECs of quiescent mature blood vessels of normal adult tissues. These rapidly proliferating ECs are vulnerable to cytotoxic/static agents (Figure 2).

That anti-tumor agents have endothelial toxicity is not a very new observation $[8,9]$. A drug that requires a higher concentration to kill endothelial cells than is required to kill cancer cells should not be considered anti-angiogenic. The strength of the available data on which this claim is founded (anti-angiogenic properties of metronomic chemotherapy) varies dramatically. Is every cytotoxic agent really antiangiogenic? Most authors do not draw this conclusion. Indeed, multiple potential criteria are necessary (see Table 2) for defining chemotherapy with anticancer agents 


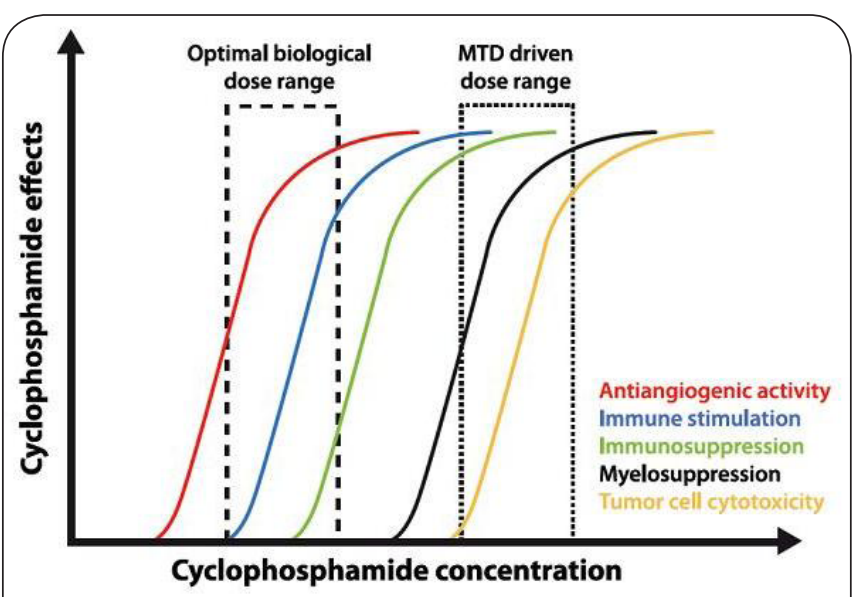

Figure 3. Dose-response curves for different effects of cyclophosphamide. Please note that these dose-effect-curves are not always overlapping. (modified from [126]).

as metronomic.

Table 2. Criteria for an antiangiogenic approach of metronomic CHT.

- Strong differential cytotoxicity between cancer cells and endothelial cells

- altered function of endothelial cells shown in dynamic contrast enhanced magnetic resonance imaging (DCEMRI) or contrast enhanced ultrasonic examinations (CEUS) by changes in the permeability and blood-flow in tumors

- changes of mechanistic effects (e.g. biomarker changes: IL-1 \&6, UPA, VEGF, VEGFR1\&2, bFGF, Ang 1\&2, MMP$2 \& 9$, vessel density etc.)

- inhibition of angiogenesis in vivo \& in vitro (in vivo models at best only with spontaneous, slow growing tumors)

Although we might think of anti-angiogenic activity as being mediated by direct effects on endothelial cells, it is equally possible for an anti-angiogenic activity to be mediated by cancer cells. If cancer cells produce proangiogenic factors in an autocrine fashion, which is the driving force behind angiogenesis, then killing cancer cells will effect endothelial cells just by eliminating these survival factors critical to endothelial cells. It would be trivial to postulate that every successful cytotoxic therapy is anti-angiogenic because in each NED (no evidence of disease) resp. CR (complete remission) stage the tumor has diappeared or not visible, but also the tumor's vasculature now is absent resp. is no longer visible anymore. Greater awareness of the collateral damaging effects of most anticancer therapeutics on the micro-environment of cancer cells should indeed make it possible to optimise this beneficial therapeutic side effect to minimise host toxicity (Figure 3).

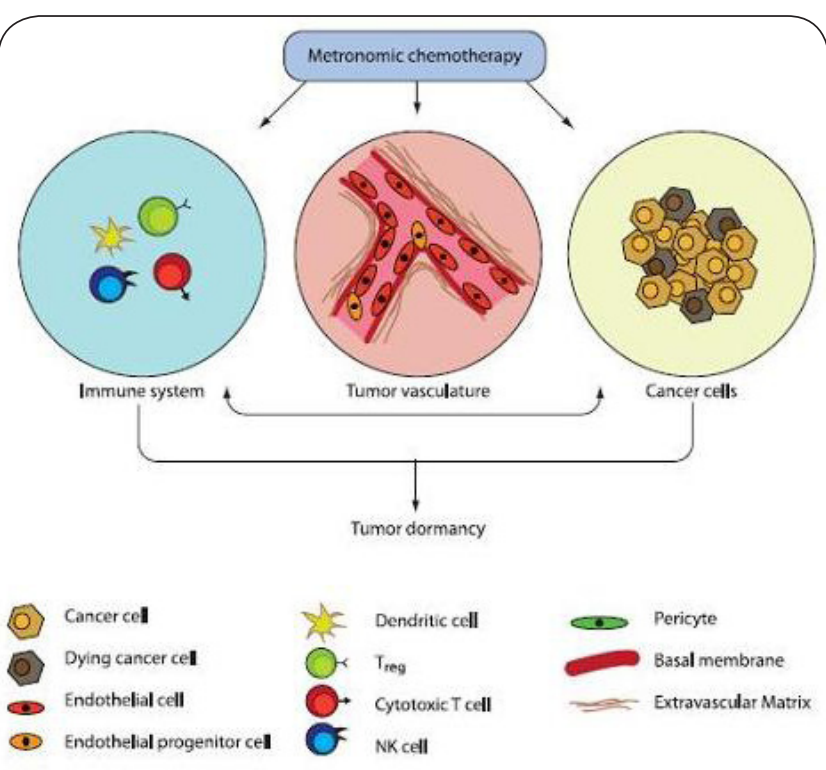

Figure 4. Different mechanisms of action of a metronomic chemotherapy. (modified from [127]).

Combination therapies of metronomic chemotherapy plus antiangiogenic drugs have shown some anti-angiogenic effects $[10,11]$, demonstrated with the available techniques from 10 to 20 years ago. The effects of cytostatic agents on endothelial cells have been described [12] and in general it is accepted that chemotherapy has, in a schedule dependant way, measurable effects on proliferating endothelial cells but the fracton of endothelial cells which is actually proliferating in the tumors vasculature is low (only 3\%). Current knowledge based on, preclinical in vitro and in vivo data as well as emerging clinical data, suggests an anti-angiogenic activity for several existing, commonly used drugs.

\section{Activation of immunity}

It is well known that cytotoxic drugs, like anthracyclines, taxanes and especially cyclophosphamide (see figure 3 ) display important immunostimulatory properties $[13,128]$. The effects on regulatory $T$ cells $\left(T_{\text {reg }}\right)$ seem to be relevant in the context of metronomic treatments. $T_{\text {reg }}$ are $C D 4+, C D$ $25+$ lymphocytes which can inhibit an anti-tumor immune response by suppressing the activity of tumorspecific and tumorunspecific effector cells. An increase of $\mathrm{T}_{\text {reg }}$ cells can be correlated with tumor progression and lack of treatment response [14]. Several studies have shown that low-dose cyclophosphamide decreases the number of $T_{\text {reg }}$ cells, inhibiting their suppressive function $[15,16]$. Another immunestimulatory effect has been demonstrated, i.e., the induction of dendritic cell maturation [17]. The promotion of dendritic cell maturation with metronomic chemotherapy in a non-toxic concentration was please observed noted and is bridging the gap between metronomic therapy 
and immunity (Figure 4). Furthermore, metronomic cyclophosphamide showed a large increase in tumorassocciated NK cells, dendritic cells, and macrophages and occurred after the onset of tumor regression in an immunocompetent, the syngeneic mouse model [128].

\section{Induction of senescence}

Lower levels of damage by chemotherapy may trigger senescence associated with antiproliferative responses without activating the cascades of caspase activity that induce cellular apoptosis. For example dosing of $250 \mathrm{nM}$ doxorubicin generates apoptosis (in prostata cells), whereas $25 \mathrm{nM}$ induces senescence [18]. Many senescence-inducing drugs generate DNA damage to produce single- and doublestrand breaks. The induction of senescence in tumors can in fact be achieved by permanently administered, lowdose schedules of cytostatics [19]. Metronomic therapies have no great capacity of inducing apoptosis, but due to their low drug levels, senescence can be one mechanism which occurs during MT. This obsrvations warrants further investigation.

Clinical trials with 'metronomic scheduling' of cytotoxic/-static drugs

According to figure 1 a metronomic chemotherapy is defined as a therapy where at each timepoint a biologically active drug concentration acts. In this scenario certain common chemotherapy protocols include metronomic components. E.g. ECF for gastric cancer uses a continuous infusion of 5-fluorouracil, the maintenance therapy of ALL contains continuous 6-mercaptopurine, even the now commonly used weekly paclitaxel therapies (instead of q3w schedules) have a 'metronomic' character as well as the Stanford V chemotherapy regimen for the treatment of Hodgkins disease with different drugs given weekly over three months in lower dosages than in the common ABVD-MOPP or BEACOPP regimens. Even the classical CMF scheme for breast cancer patients contains a 'metronomic' part with oral cyclophosphamide over 14 days albeit with a pause of one week between the treatment cycles. The original CMF scheme developed by Bonadonna with orally administered cyclophosphamide (100 mg/m ${ }^{2}$ daily for 24 days) seems to be more effective than the i.v. scheme $\left(600 \mathrm{mg} / \mathrm{m}^{2}\right.$ at day 1) which was introduced to simplify the drug schedule and to make it more convenient for patients as shown in a phase III study conducted by the EORTC [50]. The total drug amount in the original treatment protocol was more than doubled in the case of cyclophosphamide. In this case, the 'metronomic' schedule reflects a greater dose intensity and is a MTD-defined metronomic chemotherapy $\mathrm{CHT}$. In a recent published phase III study [67] it was shown in gestational trophoblastic neoplasia (GTN) that a bi-weekly i.v. administration of dactinomycin is better than a weekly im injection of methotrexate showing that this pulsed biweekly schedule can be viewed as a metronomic $\mathrm{CHT}$. In the majority of clinical trials, which are titled metronomic $\mathrm{CHT}$, cyclophosphamide (CYC) has an essential part because of its different effects mentioned peviously..

All these classical 'metronomic' CHTs chemotherapies use the maximal tolerable dose (MTD) and not a biological optimal dose (BOD). The dosing at the MTD is strongly related to the well known toxicity and therefore a metronomic chemotherapy can be MTD driven, but it can also be dosed far below the MTD, nearly at non-toxic dose levels, a dosing which is not well developed up to now because we use anticancer agents mostly at the maximum tolerated dose.

\section{Clinical studies with metronomic chemotherapy}

An increasing number of clinical studies using metronomic therapy schedules have been published in the last years. For this article a thorough literature research was performed in PubMed and Google scholar. 80 publications were found and acted as basis for the discussion in this review. Solid tumors as well as hematologic malignancies were included in this survey. The main focus was on patients with advanced, multiple metastasized a multiple pretreated solid tumors who had progressive disease and sometimes stable disease when entering the metronomic therapy. In the cases with stable disease, the goal of metronomic therapy is to maintain this status.. Therefore the entry criteria were not stringent e.g. always a progressive period before study entry. Three different metronomic schedules were of special interest:

1. metronomic CHT with cytostatics/cytotoxics,

2. the combination of cytostatics with other agents that may have anti-angiogenic effects,

3. the combination of cytostatics with targeted therapies. Besides, a short overview for metronomic therapy in the adjuvant setting was also considered interesting, albeit here the number of studies is limited [96-99].

The most commonly examinated tumor type is breast cancer. Here we found 16 studies with metronomic therapy, followed by colorectal cancer, glioblastoma, melanoma, non small cell lung cancer, prostate cancer and different histologic types of sarcomas [20-81]. But results in other tumors with a smaller number of publications such as head and neck cancer, adrenocortical carcinoma, renal cell carcinoma, neuroendocrine carcinoma and ovarian cancer have also been described [20-81]. Besides, there are increasing numbers of clinical studies who are engaged in hematologic malignancies, like different types of lymphomas including multiple myeloma. Also for children with advanced cancer and glioblastoma metronomic approaches are published. A summary of studies including indication, dosing schedule, response and description of the most special toxicities is depicted in table $\mathbf{3}$ (here is the literature cited).

Regarding the metronomic schedules for different cancer types, oral CHT drugs such as cyclophosphamide, methotrexate, trofosfamide and etoposide are the main chemotherapeutics used in these trials. Capecitabine and oral 5-FU prodrugs like UFT are also suitable agents and 
Mross et al. Journal of Cancer Therapeutics \& Research 2012,

http://www.hoajonline.com/journals/pdf/2049-7962-1-32.pdf

doi: $10.7243 / 2049-7962-1-32$

Table 3. Metronomic (low dose) chemotherapy studies

\begin{tabular}{|c|c|c|c|c|}
\hline Cancer Type & $\begin{array}{l}\text { No of } \\
\text { Pts }\end{array}$ & Results & $\begin{array}{l}\text { Toxicities } \\
\text { Grade 3/4 }\end{array}$ & Ref. \\
\hline \multicolumn{5}{|l|}{ Advanced Cancer } \\
\hline CAP 2x500 mg bid, Celecoxib 2x400 mg bid. continuously & 37 & $17 / 37$ non-PD & no & 20 \\
\hline $\begin{array}{l}\text { CYC } 50 \mathrm{mg} \text { od, Rofecoxib } 25 \mathrm{mg} \text { od, VBL } 3 \mathrm{mg} / \mathrm{m}^{2} \text { i.v. q1w, } 50 \% \text { of } \\
\text { pts received Minocycline } 100 \mathrm{mg} \text { orally bid }\end{array}$ & 47 & 14/47 non-PD & $\begin{array}{l}\text { neutropenia } 10 / 2 \\
\text { anemia } 2 / 10 \\
\text { thrombopenia } 1 / 0\end{array}$ & 21 \\
\hline $\begin{array}{l}\text { VP-16 } 25 \mathrm{mg} / \mathrm{m}^{2} \mathrm{~d} 1-14, \text { CYC } 25 \mathrm{mg} / \mathrm{m}^{2} \mathrm{~d} 15-28 \text {, Celecoxib 100-400 } \\
\mathrm{mg} / \mathrm{d} \mathrm{d} 1-28\end{array}$ & 17 & $7 / 17$ non-PD & $\begin{array}{l}\text { neutropenia } 1 / 0 \\
\text { anemia } 0 / 2 \\
\text { thrombopenia } 0 / 4\end{array}$ & 22 \\
\hline $\begin{array}{l}\text { VIN 20-50 mg thrice a week, escalated by } 10 \mathrm{mg} \text { increments in } \\
\text { successive cohorts }\end{array}$ & 62 & 25/52 non-PD & neutropenia $0 / 2$ & 23 \\
\hline TRO 50-150 mg daily & 31 & $17 / 31$ non-PD & no & 24 \\
\hline CYC average $52 \mathrm{mg}$ od continuously & 24 & TTF 6,4 months & Nausea $2 / 0$ & 25 \\
\hline $\begin{array}{l}\text { CYC } 50 \text { mg od, Celecoxib } 400 \mathrm{mg} \text { bd for } 7 \text { days each week, MTX 2,5 } \\
\text { mg bd for } 2 \text { consecutive days each week }\end{array}$ & 69 & $23 / 67$ non-PD & $\begin{array}{l}\text { Lymphopenia } 22 / 0 \\
\text { Fatigue } 11 / 0\end{array}$ & 26 \\
\hline \multicolumn{5}{|l|}{ Pediatric cancer advanced } \\
\hline $\begin{array}{l}\text { VIN } 1,5 \mathrm{mg} / \mathrm{m}^{2} \mathrm{~d} 1,8,15,22, \text { CYC } 25 \mathrm{mg} / \mathrm{m}^{2} \text { od d1-21, MTX } 15 \mathrm{mg} / \\
\mathrm{m}^{2} \text { twice weekly d21-42, followed by a } 1 \text { week break }\end{array}$ & 12 & $7 / 12$ non-PD & $\begin{array}{l}\text { anaemia } 0 / 1 \\
\text { nonfebrile neutropenia } 0 / 1\end{array}$ & 27 \\
\hline \multicolumn{5}{|l|}{ Colorectal Cancer } \\
\hline $\begin{array}{l}\text { OXA } 85 \mathrm{mg} / \mathrm{m}^{2} \mathrm{~d} 1, \mathrm{LV} 200 \mathrm{mg} / \mathrm{m}^{2} \mathrm{~d} 1 \text {, i.v. bolus } 5 \text {-FU } 400 \mathrm{mg} / \mathrm{m}^{2} \mathrm{~d} 1 \text {, } \\
\text { 22h infusion of } 5 \text {-FU } 600 \mathrm{mg} / \mathrm{m}^{2} \mathrm{~d} 1 \text {, followed by } 10 \text { day daily oral } \\
\text { UFT } 200 \mathrm{mgm}^{2} / \mathrm{LV} 30 \mathrm{mg} / \mathrm{m}^{2} \text {, every } 2 \text { weeks }\end{array}$ & 28 & $\begin{array}{l}10 / 28 \text { non-PD } \\
\text { SD not assessed }\end{array}$ & no & 28 \\
\hline $\begin{array}{l}\text { CPT- } 11 \text { continuously } 3 \text { out of } 4 \text { weeks, escalating per cohort } 1,4 \mathrm{mg} / \\
\mathrm{m}^{2} / \mathrm{d}, 2,8 \mathrm{mg} / \mathrm{m}^{2} / \mathrm{d}, 4,2 \mathrm{mg} / \mathrm{m}^{2} / \mathrm{d}\end{array}$ & 20 & $4 / 20$ non-PD & no & 29 \\
\hline $\begin{array}{l}\text { S-1 } 80 \mathrm{mg} / \mathrm{m}^{2} \mathrm{~d} 3-7,10-14,17-21, \mathrm{CPT}-11 \text { (with escalating dosages) } \\
\text { from } 40 \mathrm{mg} / \mathrm{m}^{2} \mathrm{~d} 1,8,15 \text {, qd } 29\end{array}$ & 16 & $15 / 16$ non-PD & diarrhoea $1 / 0$ & 30 \\
\hline \multicolumn{5}{|l|}{ Glioblastoma } \\
\hline $\begin{array}{l}\text { VP-16 } 35 \mathrm{mg} / \mathrm{m}^{2} \mathrm{~d} 1-21 \text {, alternating every } 21 \text { days with CYC } 2 \mathrm{mg} / \\
\mathrm{kg} \mathrm{KG} \text {, in comb. with THAL } 50-200 \mathrm{mg} \text {, escalating up to } 1200 \mathrm{mg} \text {, } \\
\text { Celecoxib } 2 \times 200 \mathrm{mg} \text { up to } 2 \times 400 \mathrm{mg} \text {. }\end{array}$ & 48 & $31 / 48$ non-PD & $\begin{array}{l}\text { constipation } 3 / 2 \\
\text { fatigue } 1 / 0 \\
\text { leukopenia } 7 / 6 \\
\text { nausea } 4 / 0\end{array}$ & 31 \\
\hline MTX 5 mg 2x/ week, CYC 100 mg od & 10 & $10 / 10 \mathrm{PD}$ & no & 32 \\
\hline TMZ $40 \mathrm{mg} / \mathrm{m}^{2}$ od & 12 & $7 / 12$ non-PD & no & 33 \\
\hline \multicolumn{5}{|l|}{ Childhood brain tumors } \\
\hline Topotecan po $0,8 \mathrm{mg} / \mathrm{m}^{2} \mathrm{~d} 1-21, \mathrm{qd} 28$ & 26 & $6 / 26$ non-PD & $\begin{array}{l}\text { neutropenia } 6 / 4 \\
\text { leucopenia } 8 / 1 \\
\text { thrombocytopenia } 6 / 2\end{array}$ & 34 \\
\hline \multicolumn{5}{|l|}{ Head and neck cancer } \\
\hline PAC $80 \mathrm{mg} / \mathrm{m}^{2}$ i.v. $\mathrm{q} 1 \mathrm{w}$ & 33 & 20/33 non-PD & no & 35 \\
\hline \multicolumn{5}{|l|}{ Lymphoma } \\
\hline CYC 50 mg od, MTX 2,5 mg 4x/ week, Celecoxib 400 mg bid & 41 & 33/41 non-PD & $\begin{array}{l}\text { No Grading } \\
\text { fatigue } \\
\text { nausea } \\
\text { neutropenia } \\
\text { anemia }\end{array}$ & 36 \\
\hline CYC 50 mg od, Celecoxib 400 mg bid & 35 & 19/35 non-PD & $\begin{array}{l}\text { fatigue } 6 / 0 \\
\text { thrombopenia } 5 / 0 \\
\text { ASAT/ALAT - } 1 / 0\end{array}$ & 37 \\
\hline $\begin{array}{l}\text { Pred } 20 \mathrm{mg} \text {, CYC } 50 \mathrm{mg} \text {, VP-16 } 50 \mathrm{mg} \text {, PCZ } 50 \mathrm{mg} \text { od, dose } \\
\text { adjustment according to WBC count }\end{array}$ & 22 & $15 / 22$ non-PD & $\begin{array}{l}\text { G } 3+4 \text { combined } \\
\text { infection } 3 \\
\text { anemia } 1 \\
\text { thrombopenia } 1\end{array}$ & 38 \\
\hline $\begin{array}{l}\text { Pred } 20 \mathrm{mg} \text {, CYC } 50 \mathrm{mg} \text {, VP-16 } 50 \mathrm{mg} \text {, PCZ } 50 \mathrm{mg} \text { daily, dose } \\
\text { adjustment according to WBC count }\end{array}$ & 75 & $\begin{array}{l}51 / 75 \text { non-PD } \\
\text { SD not assessed }\end{array}$ & $\begin{array}{l}\text { G } 3+4 \text { combined } \\
\text { infection } 8 \text { gastrointestinal } 4 \\
\text { thrombopenia } 8\end{array}$ & 39 \\
\hline
\end{tabular}


Mross et al. Journal of Cancer Therapeutics \& Research 2012, http://www.hoajonline.com/journals/pdf/2049-7962-1-32.pdf

doi: 10.7243/2049-7962-1-32

\begin{tabular}{|c|c|c|c|c|}
\hline Cancer Type & $\begin{array}{l}\text { No of } \\
\text { Pts }\end{array}$ & Results & $\begin{array}{l}\text { Toxicities } \\
\text { Grade } 3 / 4 \\
\end{array}$ & Ref. \\
\hline $\begin{array}{l}\text { VBL } 6 \mathrm{mg} / \mathrm{m}^{2}+\text { DOX } 25 \mathrm{mg} / \mathrm{m} 2 \mathrm{w} 1,3,5,7,9,11, \mathrm{VCR} 1,4 \mathrm{mg} / \mathrm{m}^{2}+ \\
\text { BLEO } 5 \text { units } / \mathrm{m}^{2} \text { w2 } 24,6,8,10,12 \text {, mustard } 6 \mathrm{mg} / \mathrm{m}^{2} \mathrm{w} 1,5,9+\mathrm{VP}-16 \\
60 \mathrm{mg} / \mathrm{m}^{2} 2 \mathrm{x} / \text { week w3,7,11, Pred } 40 \mathrm{mg} / \mathrm{m}^{2} \text { every other day w1-10 }\end{array}$ & 142 & $126 / 142$ non-PD & $\begin{array}{l}\text { G } 3+4 \text { combined } \\
\text { neutropenia } 140 \\
\text { anemia } 44 \\
\text { thrombopenia } 1 \\
\text { fatigue } 10\end{array}$ & 40 \\
\hline \multicolumn{5}{|l|}{ Metastatic Breast Cancer } \\
\hline CYC $50 \mathrm{mg}$ od d1-21, qd29 + MA $80 \mathrm{mg}$ bid & 29 & $12 / 29$ non-PD & none & 41 \\
\hline $\begin{array}{l}\text { (Arm A):CYC } 50 \mathrm{mg} \text { od }+ \text { MTX } 2,5 \mathrm{mg} \text { bid d } 1+4(\text { Arm B):CYC } 50 \\
\text { mg od + MTX 2,5 mg bid d } 1+4 \\
+ \text { THAL } 200 \mathrm{mg} \text { od }\end{array}$ & 171 & $\begin{array}{l}\text { Arm A: } \\
\text { 36/86 non-PD } \\
\text { Arm B: } \\
35 / 85 \text { non-PD }\end{array}$ & $\begin{array}{l}\text { G } 3+4 \text { combined } \\
\text { ASAT/ALAT - } 9 \\
\text { neutropenia } 4 \\
\text { lymphopenia } 3 \\
\text { anemia } 2\end{array}$ & 42 \\
\hline $\begin{array}{l}\text { Dalteparin } 5000 \mathrm{U} \text { s.c. od + CYC } 50 \mathrm{mg} \text { od + MTX 2,5 mg bid twice } \\
\text { weekly, Pred } 5 \mathrm{mg} \text { od }\end{array}$ & 41 & 10/41 non-PD & ASAT/ALAT - 11/0 & 43 \\
\hline Letrozole plus/minus CYC $50 \mathrm{mg}$ od & 114 & $\begin{array}{l}\text { LET } \\
\text { 53/57 non-PD } \\
\text { LET - CYC } \\
\text { 54/57 non-PD }\end{array}$ & $\begin{array}{l}\mathrm{CHF} 0 / 3 \\
\text { atrial flutter } 1 / 0 \\
\text { skeletal fractures } 3 / 0 \\
\text { (Causing } 1 \text { death) }\end{array}$ & 44 \\
\hline MTX 2,5 mg bid d1+2 each week + CYC $50 \mathrm{mg}$ od & 64 & 22/63 non-PD & ASAT/ALAT - 9/0 & 45 \\
\hline MTX 2,5 mg d1+2 each week + CYC $50 \mathrm{mg}$ od & 42 & $13 / 42$ non-PD & $\begin{array}{l}\text { neutropenia 3/1 } \\
\text { ASAT/ALAT - 2/0 }\end{array}$ & 46 \\
\hline $\begin{array}{l}\text { VIN } 70 \mathrm{mg} / \mathrm{m}^{2} \mathrm{~d} 1,3,5 \\
3 \text { weeks on } 1 \text { week off }\end{array}$ & 34 & 24/34 non-PD & $\begin{array}{l}\text { neutropenia } 3 / 0 \\
\text { anemia } 3 / 0 \\
\text { thrombopenia } 1 / 0\end{array}$ & 47 \\
\hline TRO $3 \times 50 \mathrm{mg}$ daily or TRO $3 \times 150 \mathrm{mg}$ d1-10, qd28 & 53 & 20/53 non-PD & $\begin{array}{l}\text { leukopenia } 4 / 0 \\
\text { thrombopenia } 0 / 2 \\
\text { anemia } 3 / 3 \\
\text { nausea } 4 / 1\end{array}$ & 48 \\
\hline $\begin{array}{l}\text { Arm A: PAC } 175 \mathrm{mg} / \mathrm{m}^{2} \mathrm{~d} 1, \mathrm{q} 3 \mathrm{w}+/ \text { - trastuzumab } \\
\text { Arm B:PAC } 80 \mathrm{mg} / \mathrm{m}^{2} \text { weekly }+/ \text { - trastuzumab }\end{array}$ & 577 & $\begin{array}{l}\text { Arm A: } \\
78 / 225 \text { non-PD } \\
\text { Arm B: } \\
\text { 145/346 non-PD }\end{array}$ & $\begin{array}{l}\text { leucopenia } 38 / 9 \\
\text { thrombopenia } 7 / 2 \\
\text { anemia } 23 / 1 \\
\text { neutropenia } 41 / 23 \\
\text { neurosensory } 111 / 1 \\
\text { fatigue } 31 / 1\end{array}$ & 49 \\
\hline $\begin{array}{l}\text { Arm A: CYC } 100 \mathrm{mg} / \mathrm{m}^{2} \text { po d } 1-14+\text { MTX } 40 \mathrm{mg} / \mathrm{m}^{2} \text { iv } \mathrm{d} 1,8+5-\mathrm{FU} \\
600 \mathrm{mg} / \mathrm{m}^{2} \text { iv } \mathrm{d} 1,8, \mathrm{q} 4 \mathrm{w} \\
\text { Arm B:CYC } 600 \mathrm{mg} / \mathrm{m}^{2} \text { iv } \mathrm{d} 1+\text { MTX } 40 \mathrm{mg} / \mathrm{m}^{2} \text { iv } \mathrm{d} 1+5-\mathrm{FU} 600 \\
\mathrm{mg} / \mathrm{m}^{2} \text { iv d } 1, \mathrm{q} 3 \mathrm{w}\end{array}$ & 254 & $\begin{array}{l}\text { Arm A: } 88 / 125 \text { non- } \\
\text { PD } \\
\text { Arm B: } 61 / 129 \text { non- } \\
\text { PD }\end{array}$ & $\begin{array}{l}\text { leucopenia } 45 / 7 \\
\text { thrombopenia } 4 / 3 \\
\text { nausea } 68 / 9\end{array}$ & 50 \\
\hline $\begin{array}{l}\text { Arm A: CYC } 50 \mathrm{mg} \text { od } \\
\text { Arm B: CYC } 50 \mathrm{mg} \text { od + MTX 2,5 mg twice weekly }\end{array}$ & 61 & $\begin{array}{l}\text { Arm A: } 12 / 22 \text { non-PD } \\
\text { Arm B: } 20 / 39 \text { non-PD }\end{array}$ & $\begin{array}{l}\text { leukopenia } 2 / 0 \\
\text { thrombocytopenia } 1 / 0\end{array}$ & 51 \\
\hline $\begin{array}{l}\text { CYC } 65 \mathrm{mg} / \mathrm{m}^{2} \text { od d } 1-14+\text { Capecitabine } 1 \mathrm{~g} / \mathrm{m}^{2} \text { twice daily d } 1-14 \text {, } \\
\mathrm{qd} 21\end{array}$ & 66 & 20/66 non-PD & $\begin{array}{l}\text { Hand-foot-s. } 3 / 0 \\
\text { Anorexia } 4 \text { (grade } 3+4 \text { combined) }\end{array}$ & 52 \\
\hline $\begin{array}{l}\text { VIN po } 30 \mathrm{mg} \text { od continuously (starting dose) + Capecitabine } 800 \\
\mathrm{mg} / \mathrm{m}^{2} \text { bid (starting dose) d1-14, } \mathrm{qd} 21\end{array}$ & 36 & $\begin{array}{l}12 / 36 \text { non-PD } \\
\text { SD not documented }\end{array}$ & & 53 \\
\hline CYC 100 mg od d1-14 + CAP 1500 mg bid d 8-21, qd21 & 80 & $54 / 80$ non-PD & $\begin{array}{l}\text { leucopenia } 15 / 0 \\
\text { lymphopenia } 10 / 3\end{array}$ & 54 \\
\hline $\begin{array}{l}50 \text { mg od continuously }+ \text { MTX } 2,5 \text { mg po bid d } 2+4 \text { every week }+1 \\
\text { mg } 1 \text { E10 anti-idiotype MAb (racotumomab) s.c.bi-weekly x } 5\end{array}$ & 21 & 13/21 non-PD & nausea/vomiting $1 / 0$ & 55 \\
\hline CAP $1500 \mathrm{mg} 0 \mathrm{~d}$ & 58 & $50 / 58$ non-PD & $\begin{array}{l}\text { G } 3+4 \text { combined } \\
\text { Hand-foot-s. } 3\end{array}$ & 56 \\
\hline \multicolumn{5}{|l|}{ Melanoma } \\
\hline $\begin{array}{l}\text { Arm A } \\
\text { TRO 3x50 mg od } \\
\text { Arm B } \\
\text { TRO 3x50 mg od + Rofecoxib } 25 \mathrm{mg} / \mathrm{od}+\text { Pioglitazone } 60 \mathrm{mg} \text { od }\end{array}$ & 76 & $\begin{array}{l}\text { Arm A: } \\
\text { 2/32 non-PD } \\
\text { Arm B: } \\
6 / 35 \text { non-PD }\end{array}$ & $\begin{array}{l}\text { hematologic } 8 / 0 \\
\text { (not differentiated) }\end{array}$ & 57 \\
\hline CYC $50-100 \mathrm{mg} 3$ weeks out of 4 & 13 & 6/13 non-PD & $\begin{array}{l}\text { G } 3+4 \text { combined } \\
\text { lymphopenia } 5 \\
\text { neutropenia } 2\end{array}$ & 58 \\
\hline
\end{tabular}


Mross et al. Journal of Cancer Therapeutics \& Research 2012,

http://www.hoajonline.com/journals/pdf/2049-7962-1-32.pdf

doi: $10.7243 / 2049-7962-1-32$

\begin{tabular}{|c|c|c|c|c|}
\hline Cancer Type & $\begin{array}{l}\text { No of } \\
\text { Pts }\end{array}$ & Results & $\begin{array}{l}\text { Toxicities } \\
\text { Grade 3/4 } \\
\end{array}$ & Ref. \\
\hline $\begin{array}{l}\text { PAC } 10 \mathrm{mg} / \mathrm{m}^{2} 96 \text { hours weekly ci, } \\
\text { Celecoxib } 400 \mathrm{mg} \text { bid }\end{array}$ & 20 & 4/20 Non-PD & $\begin{array}{l}\text { G } 3+4 \text { combined } \\
\text { DVT/PE } 3 \\
\text { anemia } 1 \\
\text { sepsis } 1\end{array}$ & 59 \\
\hline TREO $500 \mathrm{mg}$ od, Rofecoxib $25 \mathrm{mg}$ od & 12 & 5/12 non-PD & no & 60 \\
\hline
\end{tabular}

Adrenocortical Carcinoma

GEM $800 \mathrm{mg} / \mathrm{m}^{2} \mathrm{~d} 1+8$, qd21, i.v. 5-FU $200 \mathrm{mg} / \mathrm{m}^{2} / \mathrm{d}$ continuously without interruption in first 6 patients, CAP $1500 \mathrm{mg} / \mathrm{d}$ in subsequent patients. Mitotane for all Patients.

$28 \quad 13 / 28$ non-PD

Neuroendocrine carcinomas

5-FU iv $200 \mathrm{mg} / \mathrm{m}^{2}$ continuously+ LAR Ocreotide $20 \mathrm{mg} \mathrm{q} 4 \mathrm{w}$

27/29 non-PD

Non small cell lung cancer

VIN 40-70 mg trice per week, CDDP 70-85 mg/m² i.v. d1 q3w

$15 / 24$ non-PD

DOC $25 \mathrm{mg} / \mathrm{m}^{2} \mathrm{~d} 1,8,15$, qd29, TRO $50 \mathrm{mg}$ od

CDDP $30 \mathrm{mg} / \mathrm{m}^{2} \mathrm{~d} 1,8,14,28+\mathrm{VP}-1650 \mathrm{mg} / \mathrm{m}^{2} \mathrm{~d} 1-21, \mathrm{qd} 29$

$18 / 31$ non-PD

\section{Ovarian Cancer}

Case Report

CYC $50 \mathrm{mg}$ od

non-PD

Arm A: PAC $180 \mathrm{mg} / \mathrm{m}^{2} \mathrm{~d} 1$ + carboplatin AUC $6 \mathrm{~d} 1$, qd21

Arm B: PAC 80 mg/m² d1,8,15 + carboplatin AUC 6 d1, qd21

Arm A:

114/135 non-PD

Arm B:

125/147 non-PD

Gestational Trophoblastic Neoplasia

Arm A: MTX 30 mg/m² IM bi-weekly

Arm B: Dactinomycin 1,25 mg/m² i,v, beweekly

Multiple Myeloma

CYC 50 mg bid for 21 days, THAL 200 mg/d, Pred 50 mg od. qd29

30/37 non-PD

\section{Prostate Cancer}

Case Report

TRO $100 \mathrm{mg}$ od

non-PD

CYC 100-150 mg per day alternately.

Mesna $400 \mathrm{mg}$ od for three weeks followed by one week of rest

$8 \quad 5 / 8$ non-PD

CYC $50 \mathrm{mg} / \mathrm{m}^{2} / \mathrm{d}$ od

$80 \quad 26 / 80$ non-PD

SD not assessed

CYC $50 \mathrm{mg}$ od, DEXA $1 \mathrm{mg}$ od

$179 / 17$ non-PD

CYC $500 \mathrm{mg} / \mathrm{m}^{2}$ i.v. d1, from day 2 CTX $50 \mathrm{mg}$ od, Celecoxib 200 mg bid, DEXA $1 \mathrm{mg}$ od

$289 / 28$ non-PD

Arm A:

DOC iv three escalated doses $30,40,50 \mathrm{mg} / \mathrm{m}^{2}$, Zoledronic acid 2 mg fixed dose, all q2w.

Arm A: DOC $\rightarrow$ ZOL

6/9 non-PD

Arm B: ZOL $\rightarrow$ DOC
G 3+4 combined

leukopenia 6

thrombopenia 1

mucositis 1

diarrhoea $1 / 0$

hand-foot-S. 2/0

mucositis $1 / 0$

neutropenia $3 / 5$

anemia $1 / 1$

neutropenia $4 / 0$

alopecia $5 / 0$

nausea $2 / 0$

anemia $10 / 0$

leukopenia $7 / 0$

Crea and BUN - 3/0

none

G 3+4 combined

neutropenia 562

thrombopenia 256

anemia 351

fatigue 23

neutropenia $3 / 1$

thrombopenia $1 / 0$

anemia $6 / 0$

leukopenia $10 / 5$

febr. neutrop. $4 / 0$

hyperglycemia $5 / 2$

sensory neuropathy $4 / 0$

none

G 2+3 combined neutropenia 8

lymphopenia 19/0

anemia $1 / 0$

leukopenia $1 / 0$

none

none

anemia $3 / 0$ 


\begin{tabular}{|c|c|c|c|c|}
\hline Cancer Type & $\begin{array}{l}\text { No of } \\
\text { Pts }\end{array}$ & Results & $\begin{array}{l}\text { Toxicities } \\
\text { Grade } 3 / 4\end{array}$ & Ref. \\
\hline CYC 50 mg od, DEXA $1 \mathrm{mg}$ od & 34 & $\begin{array}{l}\text { PSA-Response } \\
\text { 24/34 non-PD }\end{array}$ & $\begin{array}{l}\text { Grading and Number of pts. not } \\
\text { differentiated } \\
\text { anemia } \\
\text { neutropenia } \\
\text { flu-like symptoms } \\
\text { gastrointestinal S. }\end{array}$ & 76 \\
\hline CYC $50 \mathrm{mg}$ od + MTX 2,4 mg po twice weekly & 58 & $\begin{array}{l}\text { PSA- Response } \\
15 / 58 \text { non-PD } \\
\text { Measurable disease } \\
7 / 17\end{array}$ & $\begin{array}{l}\text { leukopenia } 4 / 0 \\
\text { thrombocytopenia } 2 / 0\end{array}$ & 77 \\
\hline \multicolumn{5}{|l|}{ Renal cell carcinoma } \\
\hline $\begin{array}{l}\text { Etoricoxib } 60 \mathrm{mg} \text { od + Pioglitazone } 60 \mathrm{mg} \text { od + Interferon- } \alpha 4,5 \mathrm{MU} \\
\mathrm{sc} \text { three times a week + Capecitabine } 1 \mathrm{~g} / \mathrm{m}^{2} \text { twice daily for } 14 \text { days, } \\
\mathrm{qd} 21\end{array}$ & 45 & 16/45 non-PD & $\begin{array}{l}\text { hand-foot-s. } 16 / 0 \\
\text { diarrhea } 4 / 0 \\
\text { pneumonia } 2 / 0\end{array}$ & 78 \\
\hline \multicolumn{5}{|l|}{ Malignant vascular tumors } \\
\hline $\begin{array}{l}\text { Pioglitazone } 45 \mathrm{mg} \text { od, Rofecoxib } 25 \mathrm{mg} \text { od, after } 14 \text { days TRO 3x50 } \\
\text { mg od }\end{array}$ & 6 & $6 / 6$ non-PD & none & 79 \\
\hline \multicolumn{5}{|l|}{ Sarcomas } \\
\hline Pioglitazone $45 \mathrm{mg} /$ od, Rofecoxib $25 \mathrm{mg}$ od, TRO 3x50 mg od & 1 & non-PD & none & 80 \\
\hline VP-16 po 100 mg d1-21, qd29 & 26 & $12 / 26$ non-PD & $\begin{array}{l}\text { febr. neutrop. } 0 / 2 \\
1 \text { death due to sepsis }\end{array}$ & 81 \\
\hline \multicolumn{5}{|l|}{ Sarcoma and melanoma } \\
\hline $\begin{array}{l}\text { Pioglitazone } 45 \mathrm{mg} \text { od + Rofecoxib } 25 \mathrm{mg} \text { od + after } 14 \text { days TRO } \\
3 \times 50 \mathrm{mg} \text { od }\end{array}$ & 40 & $11 / 40$ non-PD & none & 82 \\
\hline \multicolumn{5}{|l|}{ Esophagogastric Cancer } \\
\hline $\begin{array}{l}\text { Arm A: EPI } 50 \mathrm{mg} / \mathrm{m}^{2} \mathrm{~d} 1+\text { CDDP } 60 \mathrm{mg} / \mathrm{m}^{2} \mathrm{~d} 1, \mathrm{q} 3 \mathrm{w}, 5-\mathrm{FU} 200 \\
\mathrm{mg} / \mathrm{m}^{2} / \mathrm{d} \mathrm{d} 1-21 \\
\text { Arm B: MMC } 7 \mathrm{mg} / \mathrm{m}^{2} \mathrm{~d} 1, \mathrm{q} 6 \mathrm{w},+ \text { CDDP } 60 \mathrm{mg} / \mathrm{m}^{2} \mathrm{~d} 1, \mathrm{q} 3 \mathrm{w}+5-\mathrm{FU} \\
300 \mathrm{mg} / \mathrm{m}^{2} / \mathrm{d} \mathrm{d} 1-21\end{array}$ & 580 & $\begin{array}{l}\text { Arm A: } 182 / 284 \\
\text { non-PD } \\
\text { Arm B: } 188 / 276 \text { non- } \\
\text { PD }\end{array}$ & $\begin{array}{l}G 3+4 \text { combined } \\
\text { leucopenia } 61 \\
\text { neutropenia } 142 \\
\text { anemia } 49 \\
\text { thrombopenia } 50 \\
\text { nausea } 54 \\
\text { fatigue } 87\end{array}$ & 83 \\
\hline
\end{tabular}

Abbreviations: CAP=Capecitabin, $\mathrm{CYC}=$ Cyclophosphamide, VP-16= Vepesid, VIN= Vinorelbine, TRO= Trofosfamide, $\mathrm{OXA}=$ Oxaliplatin, 5-FU= 5-Fluorouracil, LV= Leucovorin, CPT-11= Irinotecan, THAL= Thalidomide, MTX= Methothrexate, $\mathrm{TMZ}=$ Temozolomide, $\mathrm{PAC}=$ Paclitaxel, $\mathrm{PCZ}=$ Procarbazin, $\mathrm{DOX}=$ Doxorubicin, $\mathrm{BLEO}=$ Bleomycin, $\mathrm{VCR}=\mathrm{Vincristin}, \mathrm{MA}=\mathrm{Megestat}$, $\mathrm{TREO}=$ Treosulfan, GEM= Gemcitabin, $\mathrm{CDDP}=$ Cisplatin, $\mathrm{DOC}=$ Docetaxel, $\mathrm{EPI}=$ Epirubicin, od= once daily, twice daily, $\mathrm{DEXA}=$ Dexamethason, $\mathrm{MMC}=$ Mitomycin, non-PD = no progressive disease, 1qw weekly, 2qw = bi-weekly, 3qw=every three weeks, bid $=$ bis in die (twice a day)

can be combined with other cytotoxic drugs as an oral formulation for metronomic CHT.

We found a very interesting study with capecitabine for patients with advanced breast cancer [56]. The study included 60 patients, $25 \%$ heavily pretreated with three or more prior chemotherapies and $22 \%$ had capecitabine previously as palliative treatment with intermittand capecitabine $2000 \mathrm{mg} / \mathrm{m}^{2}$ on days 1-14 every three weeks and vinorelbine $60 \mathrm{mg} / \mathrm{m}^{2}$ day $1+8$ every three weeks. The overall response rate was $24 \%$, a disease stabilization was documented in $62 \%$. Also the 13 patients who were previously treated with intermittend capecitabine had substantial benefit from the metronomic therapy with $1 \mathrm{CR}, 1 \mathrm{PR}$ and $7 \mathrm{SD}$. This is a study demonstrating safety and efficacy of metronomic capecitabine in the palliative setting with a special focus on the quality of life in advanced

\section{breast cancer.}

Cyclophosphamide is predominantly used for metronomic concepts in breast cancer, prostate cancer and lymphoma, often combined with methotrexate. Nearly all studies combine metronomic CHT with an agent that interacts with a specific target. In our survey we found combinations with oral drugs such as thalidomide, letrozole, megestrole acetate, dexamethasone, prednisone and cyclooxygenase2-inhibitors, e.g. celecoxib, rofecoxib and etoricoxib as well as the PPARy-agonist pioglitazone. Dalteparin administered subcutaneously was combined with low dose cyclophosphamide, MTX and prednisone for patients with advanced breast cancer. Interferon-a was combined with etoricoxib, pioglitazone and capecitabine in advanced renal cell carcinoma. The underlying rationale is to enhance the therapeutic effect of the low dose/dense cytotoxic regimens. 
Mross et al. Journal of Cancer Therapeutics \& Research 2012,

Furthermore, known cytotoxic/-static drugs which are only available for intravenous application are of current interest. In colorectal cancer patients drugs such as irinotecan were given in a metronomic fashion as low dose continuous infusion for 3 weeks (1,4 to $4,2 \mathrm{mg} / \mathrm{d})$ followed by a 1 week rest [29]. The treatment was well tolerated without toxicity and resulted in a disease stabilization of $20 \%$. The combination of low-dose irinotecan in a weekly schedule with $\mathrm{S} 1$ showed remarkable results with an overall response rate of $43,5 \%$ and a disease stabilization of $37,5 \%$ with low toxicity. For example only 1 Grade 3 diarrhea being observed. Other intravenous applicable drugs suitable for metronomic scheduling are paclitaxel given as a weekly infusion, in this case used for patients with advanced head and neck [35], breast [49] and ovarian cancer [66]. Vinorelbine is applied weekly for patients with advanced breast cancer. Weekly docetaxel is more favourable in combination with daily trofosfamide for advanced non small cell lung cancer although docetaxel in a weekly i.v. schedule was inferior in breast cancer and prostate cancer in comparison to a q3w schedule.

Improvement of a metronomic $\mathrm{CHT}$ in terms of tumor stabilization or prolonged progression free survival may be reached by the combination of one or two oral cytotoxic drugs with an anti-angiogenic drug such as an anti-VEGFR-2 antibody or a small molecule multi-targeted VEGFR-2 antagonist receptor tyrosine kinase inhibitor. A summary of studies is depicted in table 4.

Two studies examined be-weekly bevacizumab in combination with low dose oral cytostatic agents for breast cancer, in three studies this schedule was used in ovarian cancer. Imatinib in combination with conventional antitumor drugs was investigated in patients with hepatocellular carcinoma [90]. A combination with lapatinib was found in a case report of a patient with advanced breast cancer [91]. Weekly rituximab was combined with four oral antiproliferative oral drugs as metronomic treatment for mantle cell lymphoma [92]. Two clinical trials evaluated the effectiveness of sorafenib when combined with a low dose cytotoxic agent $[93,94]$. The indications were hepatocellular and renal cell carcinoma. Trastuzumab shows antiangiogenic effects in a metronomic schedule for advanced breast cancer patients when combined with cyclophosphamide and methotrexate [95].

Interestingly, there are three clinical studies which examined the effectiveness of metronomic therapy in the adjuvant setting, and one study which examined metronomic therapy in the neoadjuvant setting (Table 5) has been reported.

The first one was performed in patients with colorectal cancer: CPT-11 was given 3 times weekly, followed by one week rest. The combined drug for the metronomic schedule was UFT given daily, 6 month.. in combination with CPT-11 afterwards followed by six months daily as monotherapy. In another trial, patients with glioblastoma received temozolomide after standard radiochemotherapy in two different schedules: a dose dense and metronomic regimen until tumor progression. The third study examined the effectiveness of vaccination therapy combined with external beam radiation in prostate cancer patients. The urgent demand of large randomized studies is obvious otherwise no acceptance of such a treatment strategy can be expected.

In the study with neoadjuvant therapy for breast cancer the conventional schedule with doxorubicin and cyclophosphamide every three weeks was compared with the weekly application of these drugs and G-CSF support. The primary outcome was microscopic pathologic complete response $(p C R)$ at surgery.

Analyzing all studies depicted in table 1-3, the number of patients included in a study varies from 1 to 631 . Fifty-five studies included only $\leq 50$ patients. We defined responders as having either complete remission, partial remission or stable disease. This is different from normal, when responders are only patients with partial and complete response. The judgement NC (no change) can be a minor regression (less than $30 \%$ ) or a small progression (less than $20 \%$ ). The response is described as the absolute number out of the total number of patients evaluated. The results are as follows: in 74 trials, patients responded to therapy. Only one study resulted in progressive disease. 2 twoarm studies resulted in progressive disease and response in either of the treatment arms. The first was a study for patients with advanced melanoma. Treatment arm A consisted of trofosfamide $3 \times 50 \mathrm{mg} / \mathrm{d}$ continuously, arm $B$ included the same regimen plus rofecoxib $25 \mathrm{mg} / \mathrm{d}$ and pioglitazone $60 \mathrm{mg} / \mathrm{d}$ [56]. Response was documented in the combination arm as benefit in progression free survival. The second two-arm study was performed with patients with advanced prostate cancer. Treatment consisted of docetaxel in escalating doses and zoledronic acid ( $2 \mathrm{mg}$ fixed dose), every two weeks in 2 different sequences [74]. Treatment with zoledronic acid upfront followed by docetaxel showed no response.

A study evaluating the effectiveness of metronomic therapy in the adjuvant setting in patients with colorectal cancer resulted in non-inferiority for 5 -year overall survival. The adjuvant treatment with temozolomide for patients with glioblastoma consisted of two arms: temozolomide administered as a dose dense and a metronomic schedule. Here the results were in favour of the dose dense treatment arm. For prostate cancer patients the adjuvant treatment consisted of vaccination therapy with interleukin 2 before and after definitive external beam radiation therapy and resulted in the induction of prostate-specific immune responses.

Neoadjuvant treatment of inflammatory or locally advanced breast cancer with a metronomic therapy showed - in comparison to the conventional schedule - a higher pCR-rate. Further studies with a metronomic approach 
Table 4. Metronomic chemotherapy plus targeted therapies.

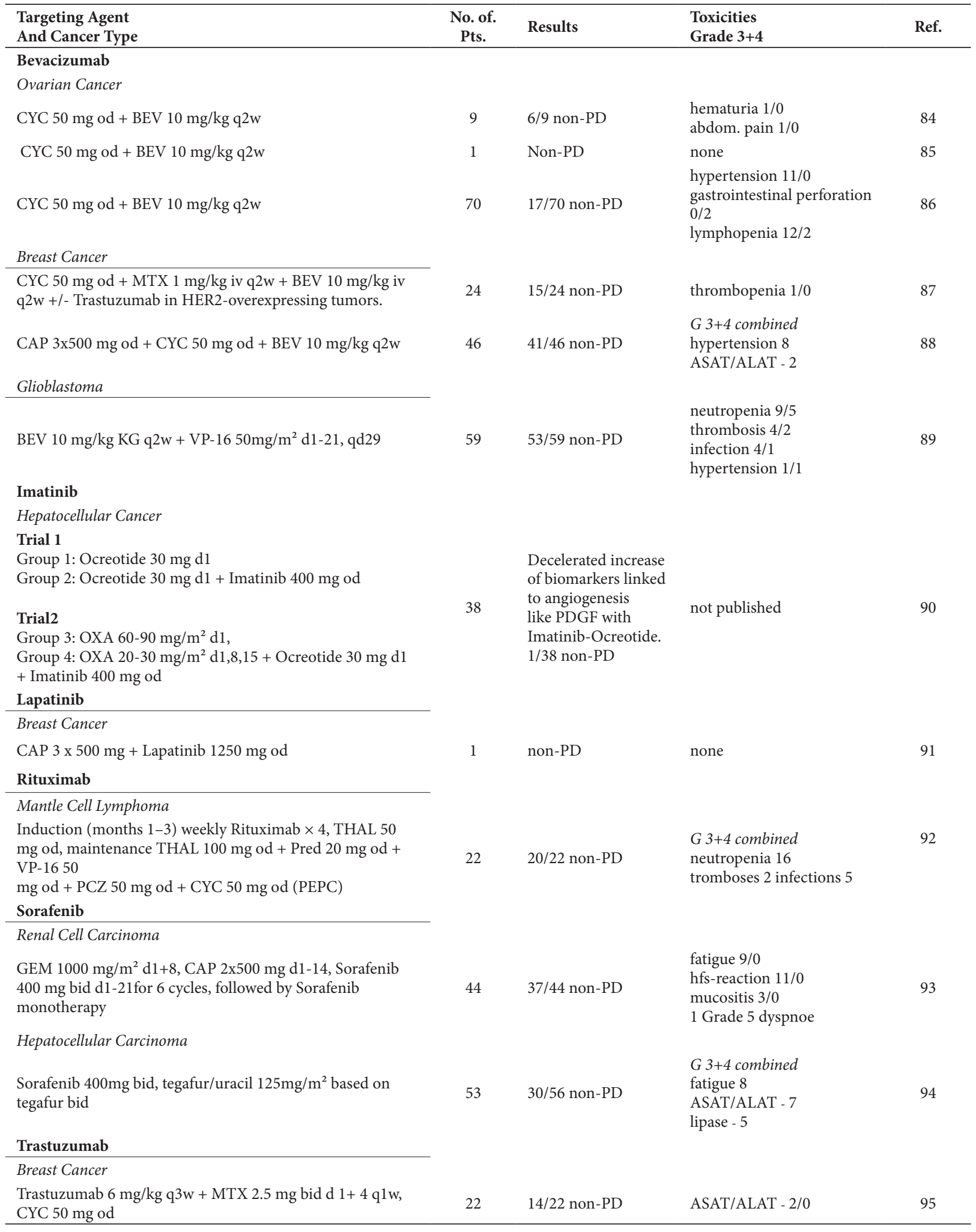

Abbreviations: $\mathrm{CYC}=$ Cyclophosphamide, $\mathrm{BEV}=$ Bevacizumab, $\mathrm{MTX}=$ Methothrexate, $\mathrm{CAP}=$ Capecitabine,

VP-16=Etoposide, $\mathrm{OXA}=$ Oxaliplatin, $\mathrm{THAL}=$ Thalidomide, $\mathrm{Pred}=$ Prednisone, $\mathrm{PCZ}=$ Procarbazine, $\mathrm{GEM}=\mathrm{Gem}$ citabine 
Table 5. Metronomic chemotherapy in adjuvant trials

\begin{tabular}{|c|c|c|c|c|}
\hline Cancer Type & $\begin{array}{c}\text { No. of. } \\
\text { Pts }\end{array}$ & Results & $\begin{array}{l}\text { Toxicities } \\
\text { Grade } 3+4\end{array}$ & Ref. \\
\hline \multicolumn{5}{|l|}{ Colorectal Cancer } \\
\hline $\begin{array}{l}\text { CPT- } 1140 \mathrm{mg} / \mathrm{m}^{2} \mathrm{~d} 1,8,15 \mathrm{qd} 29+335 \mathrm{mg} / \mathrm{m}^{2} / \mathrm{d} \text { UFT } \\
\text { od. Cycles were repeated for } 6 \text { months, followed by UFT } \\
\text { alone for further } 6 \text { months. }\end{array}$ & 49 & $\begin{array}{l}\text { Overall survival rate stage IIIb } \\
\text { group ( } 5 \text {-year: } 73 \%), \mathrm{n}=35 \\
\text { Distant metastases group ( } 5 \text { - } \\
\text { year: } 62 \%), \mathrm{n}=30\end{array}$ & neutropenia $2 / 0$ & 96 \\
\hline \multicolumn{5}{|l|}{ Glioblastoma } \\
\hline $\begin{array}{l}\text { Radiotherapy } \\
\text { with concurrent TMZ od followed by six adjuvant } \\
\text { cycles of either dose-dense }\left(150 \mathrm{mg} / \mathrm{m}^{2} \text { days } 1 \text { to } 7 \text { and }\right. \\
15 \text { to } 21) \text { or metronomic }\left(50 \mathrm{mg} / \mathrm{m}^{2} \text { od) TMZ. }\right. \\
\text { Maintenance doses of } 13 \text {-cis-retinoic acid were then } \\
\text { administered until tumor progression }\end{array}$ & 85 & $\begin{array}{l}1 \text { year Survival Rate: } \\
\text { Dose dense Arm: } 80 \% \\
\text { Metronomic Arm: } 69 \% \\
\text { Median OS } \\
\text { Dose Dense Arm: } 17,1 \mathrm{mo} \\
\text { Metronomic Arm: } 15,1 \mathrm{mo}\end{array}$ & $\begin{array}{l}\frac{G 3+4 \text { combined }}{\text { Dose dense }} \\
\text { lymphopenia } 21 \\
\text { leukopenia } 6 \\
\text { Metronomic Arm } \\
\text { lymphopenia } 17 \\
\text { ASAT/ALAT - } 5\end{array}$ & 97 \\
\hline \multicolumn{5}{|l|}{ Prostate Cancer } \\
\hline $\begin{array}{l}8 \text { planned vaccination cycles, once every } 4 \text { weeks, } \\
\text { with granulocyte-macrophage colony-stimulating } \\
\text { factor given on days } 1 \text { to } 4 \text { and interleukin } 2 \text { (IL-2) at a } \\
\text { dose of } 0.6 \mathrm{MIU} / \mathrm{M} 2 \text { given from days } 8 \text { to } 21 \text { after each } \\
\text { vaccination. Definitive external beam radiation therapy } \\
\text { was initiated after the third vaccination cycle. }\end{array}$ & 18 & $\begin{array}{l}\text { Metronomic-dose IL- } 2 \text { in } \\
\text { combination with vaccine } \\
\text { and radiation therapy is safe, } \\
\text { can induce prostate-specific } \\
\text { immune responses, and has } \\
\text { immunologic activity similar to } \\
\text { low-dose IL-2, with markedly } \\
\text { reduced toxicities. }\end{array}$ & $\begin{array}{l}\frac{\text { Grade } 3}{\mathrm{n}=\text { no of cycles }} \\
\text { fatigue } 7 \\
\text { hyperglycemia } 7 \\
\text { lymphopenia } 10\end{array}$ & 98 \\
\hline \multicolumn{5}{|l|}{ Breast Cancer } \\
\hline $\begin{array}{l}\text { Arm A: DOX } 60 \mathrm{mg} / \mathrm{m}^{2}+\text { CYC } 600 \mathrm{mg} / \mathrm{m}^{2} \mathrm{~d} 1, \mathrm{q} 3 \mathrm{w}, 5 \\
\text { cycles } \\
\text { Arm B: DOX } 24 \mathrm{mg} / \mathrm{m}^{2} \text { weekly x } 15, \text { CYC } 60 \mathrm{mg} / \mathrm{m}^{2} \text { od } \\
\text { for } 15 \text { weeks, G-CSF } 5 \mu \mathrm{g} / \mathrm{kg} / \mathrm{d} \mathrm{d} 2-7 \text { each week }\end{array}$ & 356 & $\begin{array}{l}\text { pCR } \\
\text { Arm A: } 37 / 179 \\
\text { Arm B: } 43 / 177\end{array}$ & $\begin{array}{l}\text { Arm A: } \\
\text { febr.neutropenia } 10 / 2 \\
\text { leukopenia } 39 / 23 \\
\text { neutropenia } 32 / 58 \\
\text { Arm B: } \\
\text { hand-foot-skin reaction } 25 / 0 \\
\text { leucopenia } 11 / 5 \\
\text { neutropenia } 14 / 15 \\
\text { stomatitis } 19 / 1\end{array}$ & 99 \\
\hline
\end{tabular}

Abbreviations: $\mathrm{TMZ}=$ Temozolomide, $\mathrm{CPT}-11=$ Irinotecan

are necessary to elucidate if these interesting results are reproducible.

\section{Toxicity of metronomic chemotherapy}

The patient populations, drug combinations as well as tumor types investigated thus far are very heterogenous as are the efficacy data which were reported. In total, metronomic CHT alone or in combination demonstrated a good tolerability when daily given, so this is obligatory. Most common side effects were grade 1 nausea and grade 1 to 2 anemia and neutropenia as well as grade 1 to 2 fatigue. But a severe problem is the absence of large phase III studies comparing $\mathrm{CHT}$ against metronomic $\mathrm{CHT}$. The number of treated patients with sampled toxicity data is low because phase II studies include only a small number of patients in general far below a hundred patients. Overall, metronomic CHT has often been described with minimal toxicity offering a significant benefit for the patients including quality of life. Theoretically high cumulation over time of etoposide, temozolomide and cyclophosphamide can lead to secondary leukemia, myelodysplastic syndromes (MDS) or resistance. Furthermore, if anti-angiogenic drugs are added to a metronomic $\mathrm{CHT}$, it is unclear how to dose these drugs. All approved anti-angiogenic drugs have a distinct side effect pattern, some of them have an even broader spectrum of unpleasant effects. Metronomic CHT without major side effects can easily become toxic by the addition of modern targeted drugs. Especially the difficulty to treat a common toxicity like fatigue can become a problem, as well as gastrointestinal symptoms e.g. nausea, vomiting and diarrhea. Antagonists for acute toxicity are available, but treating chronic symptoms may be much more difficult. The potential advantage of anticancer therapies with less side effects than the common MTD-driven CHT turns to a disadvantage when a toxicity is daily present.

The main toxicities Grade 3 and 4 described in all studies are depicted in table 1-3. We selected the most important side effects according to their relevance in clinical practice. Although metronomic treatment concepts use low doses of cytostatics, this therapy is not without side effects (Table 6). In 21 studies neutropenia Grade 3+4 was documented. In 9 studies elevated liver enzymes were seen. 
Mross et al. Journal of Cancer Therapeutics \& Research 2012,

http://www.hoajonline.com/journals/pdf/2049-7962-1-32.pdf

Table 6. Toxicities grade 3 and 4 of metronomic chemotherapy

\begin{tabular}{lccc}
\hline Toxicities Grade 3+4 & $\begin{array}{c}\text { Number of } \\
\text { studies }\end{array}$ & $\begin{array}{c}\text { Number of } \\
\text { patients abs. }\end{array}$ & $\begin{array}{c}\text { Number of } \\
\text { patients in \% }\end{array}$ \\
\hline Leukopenia & 15 & 320 & $5,9 \%$ \\
Lymphopenia & 8 & 124 & $2,3 \%$ \\
Neutropenia & 21 & 1122 & $20,6 \%$ \\
Anemia & 17 & 518 & $9,5 \%$ \\
Thrombopenia & 19 & 362 & $6,7 \%$ \\
Fatigue & 10 & 194 & $3,6 \%$ \\
Nausea & 9 & 215 & $3,9 \%$ \\
ASAT/ALAT - & 9 & 48 & $0,9 \%$ \\
\hline
\end{tabular}

Eight studies documented lymphopenia, 17 showed anemia, thrombocytopenia was seen in 19 and fatigue in 10 trials. In summary, 5491 patients out of all studies were treated. Regarding the absolute number of patients experiencing toxicity, the most frequent side effect is neutropenia in 1122 patients $(20,6 \%)$. Anemia is the second most frequently listed side effect, documented in 518 patients $(9,5 \%)$ but was never an indication for an interruption or termination of a metronomic therapy. Elevation of liver enzymes was seen in 48 patients, that is $0,9 \%$ of all patients assessed in the studies for this review.

\section{Metronomic therapies with non-cytotoxic/-static drugs}

Many compounds and drugs which are already approved and on the market, but not listed for anti-cancer treatment, were screened for their anti-angiogenic and anti-cancer properties and there are some interesting drugs for combinations in metronomic schedules. COX-2 is the inducible form of the enzyme that metabolizes the lipid arachidonic acid to prostaglandin $\mathrm{H} 2$, which is the first step of prostaglandin production. These compounds are widely in use as analgetic and anti-inflammatory drugs. The anti-angiogenic activity of COX-2 inhibitors is well known (100). It has been shown that the additional effects of the COX-2 inhibitors on apoptosis were distinct from their effects on COX-2 inhibition [101], but both effects are very welcome for an anti-cancer treatment approach. In general, COX-2 is up-regulated during development of cancer from early hyperplasia to metastatic cancer, especially in colon cancer, but in other cancer types as well. COX-2 was consistently and more intensively observed in metastatic lesions compared with the primary tumor. COX-2 was detected in non-cancerous cells immediately adjacent to tumor cells and in the angiogenic vasculature of the tumor, but not in the vasculature of normal tissues [102]. Furthermore, COX-2 inhibitors like celecoxib increase the intracellular accumulation of doxorubicin and enhance the cytotoxic effects via NF-kB [103]. In the adenoma prevention with celexoxib (APC) study, it was shown that celexocib can reduce the development of adenomas in a dose dependent manner [104]. It is a long known und recently updated fact that the daily intake (a metronomic schedule) of low-dose aspirin can prevent colon cancer [105]. Another interesting and novel drug for treating or preventing malignant diseases which is coming into focus is metformin. It is widely used in the treatment of diabetes mellitus type 2 to reduce insulin resistance. Population studies have suggested that metformin used in diabetic patients decreases cancer incidence and mortality. In a recent retrospective study it was found that breast cancer patients with diabetes receiving metformin have a significant higher $\mathrm{pCR}$ rate after neoadjuvant chemotherapy than patients not receiving metformin [106]. The mechanism of this effect is a matter of discussion and ongoing studies. Two important pathways are being discussed that are involved in cancer growth. One is the insulin/insulin-like growth factor-1 (IGF-1) signaling pathway which is activated when nutritients are available; the other is the adenosine mono-phosphate-acitvated protein kinase (AMPK) pathway, activated when cells are starved for carbohydrates [107]. Furthermore, low-dose metformin in combination with cyctostatic/-toxic agents like doxorubicin is able to kill cancer stem cells [108] and metformin is able to inhibit mTOR [109] as this is the effect of the activation of the AMP-activated protein kinase (AMPK) induced by metformin. The facts about this anti-diabetic drug related to cancer control have been recently reviewed and summarized [110]. In our opinion this topic is a very exciting one and has led to different clinical trials, including translational research to explore this very old and inexpensive drug in the oncology world. Metformin use is associated with a better survival of diabetic patients with pancreatic cancer [129]. The question is whether metformin treatment or the diabetes mellitus itself, with all the metabolic changes, is the explanation.

Many up-stream signals like epidermal growth factor receptor (EGF-R) or insulin-like growth factor receptor (IGF-R) feed into the PI3-kinase/Akt cascade (Figure 5).

The radiosensitizing effect of HIV protease inhibitors has been linked to the inhibition of phospho-Akt. A case report has been published in 1999 with a complete response of a Kaposi sarcoma which had been treated with an antiretroviral therapy [111]. Nelfinavir is a lead HIV protease inhibitor which inhibits AKT. It plays a crucial role in many pathways of growth factors and stimulates cancer growth. It would be of utmost interest to have an AKT inhibitor available just to interfere with these different pathways. The drug further induces $a G_{1}$ cell arrest and inhibits the proliferation of cancer cells in the $\mathrm{NCl} 60$ cell line panel [112]. Noteworthy, prolonged exposure rather than $C_{\max }$ are more important for tumor inhibition, suggesting a 'metronomic' schedule of this drug. One phase I study has been published with cisplatin, gemcitabine and oral nelfinavir [113]. Because there are abundant safety data on HIV protease inhibitors accumulated in thousands of patients, such a drug can easily be added to metronomic concepts introducing a new mechanism of action to such a concept [114]. 


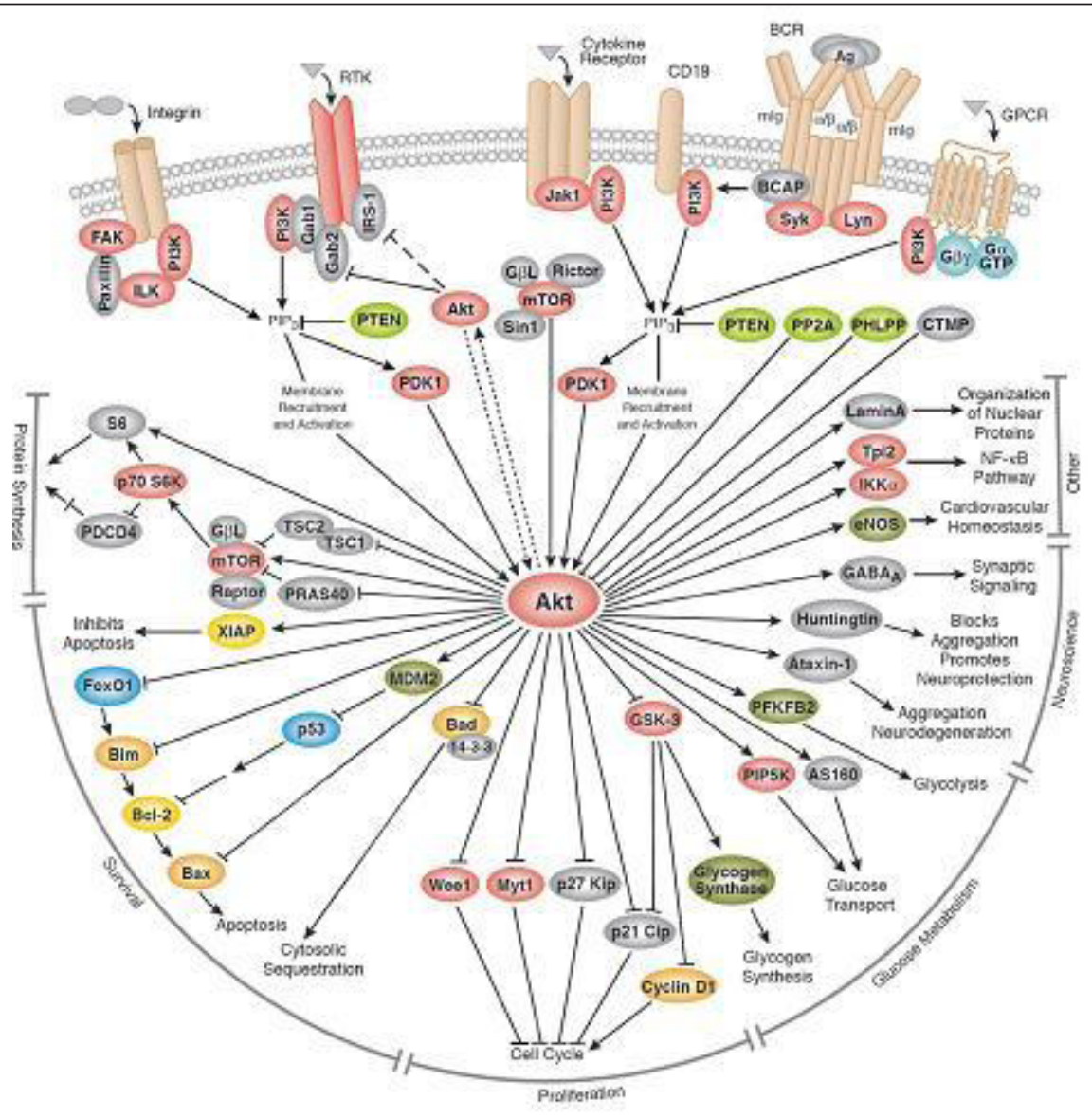

Figure 5. The central position of AKT in different pathways (from: www.cellsignal.com/reference/pathway/pdfs/Akt_PKB.pdf)

The thiazolidinediones represent another class of drugs featuring anti-neoplastic effects as peroxisome proliferatoractivated receptor $\gamma$ agonists. As cartooned in a review [115], such drugs decreased the intracellular adhesion molecule-1, the vascular cell adhesion molecule-1, increased nuclear factor KB,1 and decreased COX-2 and endothelin. PPARY ligands inhibit tumor growth and metastasis by inhibiting angiogenesis [116] because it is highly expressed in tumor endothelium in vivo and proliferating ECs in vitro. PPARY ligands inhibit CAM angiogenesis and bFGF-induced corneal neovascularisation and prevent metastatic invasion. Another mechanism described in vitro experiments is to decrease metastasis via down-regulation of AKT [117]. The PPAR represents a large family of nuclear hormone receptors of 75 proteins in the mammalian proteome that enable the cells to respond to extracellular stimuli by transcriptional regulation of gene expressions [118]. Because these drugs have an excellent side-effect profile, the addition to a metronomic chemotherapy would therefore be easy to perform. Other approved drugs like nitroxolene [119] and rifampicin [120] have been described to feature anti-angiogenic properties. Nitroxolene is approved for the prophylaxis of bladder infection, thus continuous application should not be a problem. Rifampicin enters the enterohepatic circulation and undergoes hepatic accumulation, it may be beneficial as an anti-tumor agent targeting hepatobiliary tumors. Thalidomide, a sedative with anti-angiogenic properties [121], has been used in continuous low dosages in different cancer types with some therapeutic effects [122] but also with negative results [123]. The therapeutic effects of thalidomide in multiple myeloma as well as in myelodysplastic syndromes are possibly related to the anti-angiogenic properties of this drug. Similarly, long-term treatment with daily low doses of interferon a resulted in regressions of life-threatening hemangiomas [124]. It would be an interesting task to evaluate these non-cytotoxic agents which are approved drugs (for non-cancer indications) in combination with metronomic chemotherapy as well as in combination with 'targeted drugs' because all these drugs can be orally administered in an essentially 'metronomic' way.

\section{Conclusion}

Recent advances in anti-cancer treatment have been considerable for selected solid tumors due to new anti- 
cancer drugs especially the introduction of so called 'targeted drugs' such as monoclonal antibodies and small molecule signal transduction inhibitors (-nibs), however it is noteworthy to see that we still have ample room for improving our existing therapeutic armentarium to use 'old' approved drugs in new combinations, new schedules and new dosages. The focus in the past had been to obtain cures by tumor eradication at the expense of treatment-related complications/toxicity. However, the development of metronomic anti-cancer therapies is an approach designed to maintain a stable disease situation for advanced cancer patients (patients that cannot be cured) [125]. A metronomic CHT with a single drug is highly unlikely to be of benefit to the patients and to replace the conventional MTD defined CHT protocols when rapid tumor cell killing is necessary. The fundamental truth of oncology may still apply for the different metronomic concepts described in this paper: large tumors continue to be bad tumors and rapidly proliferating tumors and a high mutation rate will limit the treatment options a medical oncologist has, either using conventional MTD driven CHT or targeted therapy or metronomic therapy. Optimizing a metronomic anticancer therapy (which drugs ?, which tumors ? and when at which time point ?) is still an open question even after a decade of clinical work. Although the background of metronomic therapy is highly interesting, there is still a lack of good to excellent clinical trials. Phase III studies have not been performed and even high quality phase II studies are rare. This is actually the biggest hurdle for this low-cost and low-toxicity concept. It has to be shown that metronomic therapy is not a disappointing low-efficacy concept which only works in few cases as a palliative treatment. It is a great challenge to show that a mixture of drugs given in a metronomic way is effective and realistic step forward in controlling cancer.

\section{Competing interests}

The authors declare that they have no competing interests.

\section{Authors' contributions}

Both authors contributed equally to this article (conception, interpretation of data, writing and final approval).

\section{Acknowledgement}

We acknowledge the corrections of the text by Abigail

Dossett and Dr. Felix Kratz and the assistance in the preparations of the modified figures by Markus Mross.

\section{Publication history}

Editor: David J. Waxman, Boston University, USA.

EIC: G.J. Peters, VU University Medical Center, Netherlands. Received: 06-Aug-2012 Revised: 25-Oct-2012

Re-revised: 20-Nov-2012 Accepted: 29-Nov-2012

Published: 13-Dec-2012

\section{References}

1. Folkman J: Tumor angiogenesis: therapeutic implications. N Engl J Med 1971, 285:1182-1186. | Article | PubMed

2. Hurwitz H, Fehrenbacher L, Novotny W, Cartwright T, Hainsworth J, Heim W, Berlin J, Baron A, Griffing S, Holmgren E, Ferrara N, Fyfe G, Rogers B, Ross R, Kabbinavar F: Bevacizumab plus irinotecan, fluorouracil, and leucovorin for metastatic colorectal cancer. N Engl J Med 2004, 350:2335-2342. | Article | PubMed

3. Miller KD, Sweeney CJ, Sledge GW, Jr.: Redefining the target: chemotherapeutics as antiangiogenics. J Clin Oncol 2001, 19:1195-1206. | Article I PubMed

4. Browder T, Butterfield CE, Kraling BM, Shi B, Marshall B, O’Reilly MS, Folkman J: Antiangiogenic scheduling of chemotherapy improves efficacy against experimental drug-resistant cancer. Cancer Res 2000, 60:1878-1886. | Article | PubMed

5. Hanahan D, Bergers G, Bergsland E: Less is more, regularly: metronomic dosing of cytotoxic drugs can target tumor angiogenesis in mice. J Clin Invest 2000, 105:1045-1047. | Article | PubMed Abstract | PubMed Full Text

6. Mross K: Anti-angiogenesis therapy:concepts and importance of dosing schedules in clinical trials. Drug Resist Updat 2000, 3:223-235. | Article | PubMed

7. Mross K. Angiogeneseinhibition in der Onkologie. Uni-Med Publisher Bremen-London-Boston 2007. | Article | PubMed Abstract | PubMed Full Text

8. Lazo JS: Endothelial injury caused by antineoplastic agents. Biochem Pharmacol 1986, 35:1919-1923. | Article | PubMed

9. Doll DC, Yarbro JW: Vascular toxicity associated with antineoplastic agents. Semin Oncol 1992, 19:580-596. I PubMed

10. Klement G, Baruchel S, Rak J, Man S, Clark K, Hicklin DJ, Bohlen P, Kerbel RS: Continuous low-dose therapy with vinblastine and VEGF receptor-2 antibody induces sustained tumor regression without overt toxicity. J Clin Invest 2000, 105:R15-24. | Article | PubMed Abstract | PubMed Full Text

11. Teicher BA, Sotomayor EA, Huang ZD: Antiangiogenic agents potentiate cytotoxic cancer therapies against primary and metastatic disease. Cancer Res 1992, 52:6702-6704. I Article I PubMed

12. de Vos FY, Willemse PH, de Vries EG, Gietema JA: Endothelial cell effects of cytotoxics: balance between desired and unwanted effects. Cancer Treat Rev 2004, 30:495-513. I Article I PubMed

13. Zitvogel L, Apetoh L, Ghiringhelli F, Kroemer G: Immunological aspects of cancer chemotherapy. Nat Rev Immunol 2008, 8:59-73. | Article | PubMed

14. Kono K, Kawaida H, Takahashi A, Sugai H, Mimura K, Miyagawa N, Omata $\mathrm{H}$, Fujii H: CD4(+)CD25high regulatory T cells increase with tumor stage in patients with gastric and esophageal cancers. Cancer Immunol Immunother 2006, 55:1064-1071. | Article I PubMed

15. Loeffler M, Kruger JA, Reisfeld RA: Immunostimulatory effects of low-dose cyclophosphamide are controlled by inducible nitric oxide synthase. Cancer Res 2005, 65:5027-5030. | Article I PubMed

16. Lutsiak ME, Semnani RT, De Pascalis R, Kashmiri SV, Schlom J, Sabzevari $\mathrm{H}$ : Inhibition of $\mathrm{CD} 4(+) 25+\mathrm{T}$ regulatory cell function implicated in enhanced immune response by low-dose cyclophosphamide. Blood 2005, 105:2862-2868. | Article I PubMed

17. Tanaka H, Matsushima H, Mizumoto N, Takashima A: Classification of chemotherapeutic agents based on their differential in vitro effects on dendritic cells. Cancer Res 2009, 69:6978-6986. | Article | PubMed Abstract | PubMed Full Text

18. Ewald JA, Desotelle JA, Wilding G, Jarrard DF: Therapy-induced senescence in cancer. J Natl Cancer Inst 2010, 102:1536-1546. | Article | PubMed Abstract | PubMed Full Text

19. Schwarze SR, Fu VX, Desotelle JA, Kenowski ML, Jarrard DF: The identification of senescence-specific genes during the induction of 
senescence in prostate cancer cells. Neoplasia 2005, 7:816-823. | Article | PubMed Abstract | PubMed Full Text

20. Steinbild S, Arends J, Medinger M, Häring, Frost A, Drevs J, Unger C, Strecker R, Hennig J, Mross K: Metronomic antiangiogenic therapy with capecitabine and celecoxib in advanced tumor patientsresults of a phase II study. Onkologie 2007, 30:629-635. | Article | PubMed

21. Young SD, Whissell M, Noble JC, Cano PO, Lopez PG, Germond CJ: Phase II clinical trial results involving treatment with low-dose daily oral cyclophosphamide, weekly vinblastine, and rofecoxib in patients with advanced solid tumors. Clin Cancer Res 2006, 12:3092-3098. | Article | PubMed Abstract | PubMed Full Text

22. Andre N, Rome A, Coze C, Padovani L, Pasquier E, Camoin L, Gentet JC: Metronomic etoposide/cyclophosphamide/celecoxib regimen given to children and adolescents with refractory cancer: a preliminary monocentric study. Clin Ther 2008, 30:1336-1340. | Article I PubMed

23. Briasoulis E, Pappas P, Puozzo C, Tolis C, Fountzilas G, Dafni U, Marselos M, Pavlidis N: Dose-ranging study of metronomic oral vinorelbine in patients with advanced refractory cancer. Clin Cancer Res 2009, 15:6454-6461. | Article | PubMed

24. Gunsilius E, Gierlich T, Mross K, Gastl G, Unger C: Palliative chemotherapy in pretreated patients with advanced cancer: oral trofosfamide is effective in ovarian carcinoma. Cancer Invest 2001, 19:808-811. | Article | PubMed Abstract | PubMed Full Text

25. Bojko P, Schimmel G, Bosse D, Abenhardt W: Metronomic oral cyclophosphamide in patients with advanced solid tumors. Onkologie 2012, 35:35-38. | Article | PubMed

26. Khan OA, Blann AD, Payne MJ, Middleton MR, Protheroe AS, Talbot DC, Taylor M, Kirichek O, Han C, Patil M, Harris AL: Continuous low-dose cyclophosphamide and methotrexate combined with celecoxib for patients with advanced cancer. Br J Cancer 2011, 104:1822-1827. | Article | PubMed Abstract | PubMed Full Text

27. Fousseyni T, Diawara M, Pasquier $\mathrm{E}$, Andre $\mathrm{N}$ : Children treated with metronomic chemotherapy in a low-income country: METRO-MALI-01. J Pediatr Hematol Oncol 2011, 33:31-34. | Article | PubMed

28. Lin PC, Chen WS, Chao TC, Yang SH, Tiu CM, Liu JH: Biweekly oxaliplatin plus 1-day infusional fluorouracil/leucovorin followed by metronomic chemotherapy with tegafur/uracil in pretreated metastatic colorectal cancer. Cancer Chemother Pharmacol 2007, 60:351-356. | Article | PubMed

29. Allegrini $G$, Falcone $A$, Fioravanti $A$, Barletta $M T$, Orlandi $P$, Loupakis F, Cerri E, Masi G, Di Paolo A, Kerbel RS, Danesi R, Del Tacca M, Bocci G: A pharmacokinetic and pharmacodynamic study on metronomic irinotecan in metastatic colorectal cancer patients. Br J Cancer 2008, 98:1312-1319. | Article | PubMed Abstract | PubMed Full Text

30. Ogata Y, Sasatomi T, Akagi Y, Ishibashi N, Mori S, Shirouzu K: Dosage escalation study of S-1 and irinotecan in metronomic chemotherapy against advanced colorectal cancer. Kurume Med J 2009, 56:1-7. | Article | PubMed

31. Kesari S, Schiff D, Doherty L, Gigas DC, Batchelor TT, Muzikansky A, O'Neill A, Drappatz J, Chen-Plotkin AS, Ramakrishna N, Weiss SE, Levy B, Bradshaw J, Kracher J, Laforme A, Black PM, Folkman J, Kieran M, Wen PY: Phase II study of metronomic chemotherapy for recurrent malignant gliomas in adults. Neuro Oncol 2007, 9:354363. | Article | PubMed Abstract | PubMed Full Text

32. Herrlinger U, Rieger J, Steinbach JP, Nagele T, Dichgans J, Weller M: UKT-04 trial of continuous metronomic low-dose chemotherapy with methotrexate and cyclophosphamide for recurrent glioblastoma. J Neurooncol 2005, 71:295-299. | Article | PubMed

33. Kong DS, Lee JI, Kim WS, Son MJ, Lim do H, Kim ST, Park K, Kim $\mathrm{JH}$, Eoh W, Nam DH: A pilot study of metronomic temozolomide treatment in patients with recurrent temozolomide-refractory glioblastoma. Oncol Rep 2006, 16:1117-1121. | Article | PubMed

34. Minturn JE, Janss AJ, Fisher PG, Allen JC, Patti R, Phillips PC, Belasco JB: A phase II study of metronomic oral topotecan for recurrent childhood brain tumors. Pediatr Blood Cancer 2011, 56:39-44. |
Article | PubMed

35. Caballero M, Grau JJ, Blanch JL, Domingo-Domenech J, Auge JM, Jimenez W, Bernal-Sprekelsen M: Serum vascular endothelial growth factor as a predictive factor in metronomic (weekly) Paclitaxel treatment for advanced head and neck cancer. Arch Otolaryngol Head Neck Surg 2007, 133:1143-1148. | Article | PubMed

36. El Bary NA, Hashem T, Metwally H, Ghany AA, El Mageed HA: A phase II study of high-dose celecoxib and metronomic, low-dose' cyclophosphamide and methotrexate in patients with relapsed and refractory lymphoma. Hematol Oncol Stem Cell Ther 2010, 3:13-18. | Article | PubMed

37. Buckstein R, Kerbel RS, Shaked $Y$, Nayar R, Foden C, Turner R, Lee $C R$, Taylor D, Zhang L, Man S, Baruchel S, Stempak D, Bertolini F, Crump M: High-Dose celecoxib and metronomic "low-dose" cyclophosphamide is an effective and safe therapy in patients with relapsed and refractory aggressive histology non-Hodgkin's lymphoma. Clin Cancer Res 2006, 12:5190-5198. | Article | PubMed

38. Coleman M, Martin P, Ruan J, Furman R, Niesvizky R, Elstrom R, George P, Leonard J, Kaufmann T: Low-dose metronomic, multidrug therapy with the PEP-C oral combination chemotherapy regimen for mantle cell lymphoma. Leuk Lymphoma 2008, 49:447-450. | Article | PubMed

39. Coleman $M$, Martin $P$, Ruan J, Furman R, Niesvizky R, Elstrom R, George P, Kaufman TP, Leonard JP: Prednisone, etoposide, procarbazine, and cyclophosphamide (PEP-C) oral combination chemotherapy regimen for recurring/refractory lymphoma: low-dose metronomic, multidrug therapy. Cancer 2008, 112:2228-2232. | Article | PubMed

40. Horning SJ, Hoppe RT, Breslin S, Bartlett NL, Brown BW, Rosenberg SA: Stanford $V$ and radiotherapy for locally extensive and advanced Hodgkin's disease: mature results of a prospective clinical trial. J Clin Oncol 2002, 20:630-637. | Article | PubMed

41. Licchetta A, Correale P, Migali C, Remondo C, Francini E, Pascucci A, Magliocca A, Guarnieri A, Savelli V, Piccolomini A, Carli AF, Francini G: Oral metronomic chemo-hormonal-therapy of metastatic breast cancer with cyclophosphamide and megestrol acetate. J Chemother 2010, 22:201-204. | Article | PubMed

42. Colleoni M, Orlando L, Sanna G, Rocca A, Maisonneuve P, Peruzzotti G, Ghisini R, Sandri MT, Zorzino L, Nole F, Viale G, Goldhirsch A: Metronomic low-dose oral cyclophosphamide and methotrexate plus or minus thalidomide in metastatic breast cancer: antitumor activity and biological effects. Ann Oncol 2006, 17:232-238. | Article I PubMed

43. Wong NS, Buckman RA, Clemons M, Verma S, Dent S, Trudeau ME, Roche K, Ebos J, Kerbel R, Deboer GE, Sutherland DJ, Emmenegger U, Slingerland J, Gardner S, Pritchard KI: Phase I/II trial of metronomic chemotherapy with daily dalteparin and cyclophosphamide, twice-weekly methotrexate, and daily prednisone as therapy for metastatic breast cancer using vascular endothelial growth factor and soluble vascular endothelial growth factor receptor levels as markers of response. J Clin Oncol 2010, 28:723-730. | Article | PubMed

44. Bottini A, Generali D, Brizzi MP, Fox SB, Bersiga A, Bonardi S, Allevi G, Aguggini S, Bodini G, Milani M, Dionisio R, Bernardi C, Montruccoli A, Bruzzi P, Harris AL, Dogliotti L, Berruti A: Randomized phase II trial of letrozole and letrozole plus low-dose metronomic oral cyclophosphamide as primary systemic treatment in elderly breast cancer patients. J Clin Oncol 2006, 24:3623-3628. | Article | PubMed Abstract | PubMed Full Text

45. Colleoni M, Rocca A, Sandri MT, Zorzino L, Masci G, Nole F, Peruzzotti G, Robertson C, Orlando L, Cinieri S, de BF, Viale G, Goldhirsch A: Low-dose oral methotrexate and cyclophosphamide in metastatic breast cancer: antitumor activity and correlation with vascular endothelial growth factor levels. Ann Oncol 2002, 13:73-80. | Article | PubMed

46. Salem DA, Gado NM, Abdelaziz NN, Essa AE, Abdelhafeez ZM, Kamel $\mathrm{TH}$ : Phase II trial of metronomic chemotherapy as salvage therapy for patients with metastatic breast cancer. J Egypt Natl Canc Inst 2008, 20:134-140. | Pdf | PubMed 
47. Addeo R, Sgambato A, Cennamo G, Montella L, Faiola V, Abbruzzese A, Capasso E, Leo L, Botti G, Caraglia M, Del Prete S: Low-dose metronomic oral administration of vinorelbine in the first-line treatment of elderly patients with metastatic breast cancer. Clin Breast Cancer 2010, 10:301-306. | Article | PubMed

48. Mross K, Rüther A, Gierlich T, Unger C: Tumor Growth Control by Oral Trofosfamide in Patients with Metastatic Breast Cancer. Onkologie 1998, 21:52-56. | Article

49. Seidman AD, Berry D, Cirrincione C, Harris L, Muss H, Marcom PK, Gipson G, Burstein H, Lake D, Shapiro CL, Ungaro P, Norton L, Winer $\mathrm{E}$, Hudis C: Randomized phase III trial of weekly compared with every-3-weeks paclitaxel for metastatic breast cancer, with trastuzumab for all HER-2 overexpressors and random assignment to trastuzumab or not in HER-2 nonoverexpressors: final results of Cancer and Leukemia Group B protocol 9840. J Clin Oncol 2008, 26:1642-1649. | Article | PubMed

50. Engelsman E, Klijn JC, Rubens RD, Wildiers J, Beex LV, Nooij MA, Rotmensz N, Sylvester R: „Classical“ CMF versus a 3-weekly intravenous $\mathrm{CMF}$ schedule in postmenopausal patients with advanced breast cancer. An EORTC Breast Cancer Co-operative Group Phase III Trial (10808). Eur J Cancer 1991, 27:966-970. | Article I PubMed

51. Gebbia V, Boussen H, Valerio MR: Oral metronomic cyclophosphamide with and without methotrexate as palliative treatment for patients with metastatic breast carcinoma. Anticancer Res 2012, 32:529-536. | Article | PubMed

52. Wang Z, Lu J, Leaw S, Hong X, Wang J, Shao Z, Hu X: An all-oral combination of metronomic cyclophosphamide plus capecitabine in patients with anthracycline- and taxane-pretreated metastatic breast cancer: a phase II study. Cancer Chemother Pharmacol 2012, 69:515-522. | Article | PubMed

53. Saridaki Z, Malamos N, Kourakos P, Polyzos A, Ardavanis A, Androulakis N, Kalbakis K, Vamvakas L, Georgoulias V, Mavroudis D: A phase I trial of oral metronomic vinorelbine plus capecitabine in patients with metastatic breast cancer. Cancer Chemother Pharmacol 2012, 69:35-42. | Article | PubMed

54. Schott AF, Barlow WE, Albain KS, Chew HK, Wade JL, 3rd, Lanier KS Lew DL, Hayes DF, Gralow JR, Livingston RB, Hortobagyi GN: Phase II trial of simple oral therapy with capecitabine and cyclophosphamide in patients with metastatic breast cancer: SWOG S0430. Oncologist 2012, 17:179-187. | Article | PubMed

55. Soriano JL, Batista N, Santiesteban E, Lima M, Gonzalez J, Garcia R, Zarza Y, Lopez MV, Rodriguez M, Loys JL, Montejo N, Aguirre F, Macias A, Vazquez AM: Metronomic Cyclophosphamide and Methotrexate Chemotherapy Combined with 1E10 Anti-Idiotype Vaccine in Metastatic Breast Cancer. Int J Breast Cancer 2011, 2011:710292. | Article | PubMed Abstract | PubMed Full Text

56. Fedele $P$, Marino A, Orlando L, Schiavone P, Nacci A, Sponziello F, Rizzo P, Calvani N, Mazzoni E, Cinefra M, Cinieri S: Efficacy and safety of low-dose metronomic chemotherapy with capecitabine in heavily pretreated patients with metastatic breast cancer. Eur J Cancer 2012, 48:24-29. | Article | PubMed

57. Reichle A, Vogt T, Coras B, Terheyden P, Neuber K, Trefzer U, Schultz E, Berand A, Brocker EB, Landthaler M, Andreesen R: Targeted combined anti-inflammatory and angiostatic therapy in advanced melanoma: a randomized phase II trial. Melanoma Res 2007, 17:360-364. | Article | PubMed

58. Borne E, Desmedt E, Duhamel A, Mirabel X, Dziwniel V, Maire C, Florin V, Martinot V, Penel N, Vercambre-Darras S, Mortier L: Oral metronomic cyclophosphamide in elderly with metastatic melanoma. Invest New Drugs 2010, 28:684-689. | Article | PubMed

59. Bhatt RS, Merchan J, Parker R, Wu HK, Zhang L, Seery V, Heymach JV, Atkins MB, McDermott D, Sukhatme VP: A phase 2 pilot trial of low-dose, continuous infusion, or „metronomic“ paclitaxel and oral celecoxib in patients with metastatic melanoma. Cancer 2010, 116:1751-1756. | Article | PubMed Abstract | PubMed Full Text

60. Spieth K, Kaufmann R, Gille J: Metronomic oral low-dose treosulfan chemotherapy combined with cyclooxygenase- 2 inhibitor in pretreated advanced melanoma: a pilot study. Cancer Chemother Pharmacol 2003, 52:377-382. | Article | PubMed
61. Sperone P, Ferrero A, Daffara F, Priola A, Zaggia B, Volante M, Santini D, Vincenzi B, Badalamenti G, Intrivici C, Del Buono S, De Francia S, Kalomirakis E, Ratti R, Angeli A, Dogliotti L, Papotti M, Terzolo M, Berruti A: Gemcitabine plus metronomic 5-fluorouracil or capecitabine as a second-/third-line chemotherapy in advanced adrenocortical carcinoma: a multicenter phase II study. Endocr Relat Cancer 2010, 17:445-453. | Article | PubMed

62. Brizzi MP, Berruti A, Ferrero A, Milanesi E, Volante M, Castiglione F, Birocco N, Bombaci S, Perroni D, Ferretti B, Alabiso O, Ciuffreda L, Bertetto O, Papotti M, Dogliotti L: Continuous 5-fluorouracil infusion plus long acting octreotide in advanced well-differentiated neuroendocrine carcinomas. A phase II trial of the Piemonte oncology network. BMC Cancer 2009, 9:388. | Article | PubMed Abstract | PubMed Full Text

63. Pallis AG, Chandrinos V, Pavlakou G, Xenidis N, Varthalitis I, Vardakis N, Vamvakas L, Kontopodis E, Rovithi M, Georgoulias V: A multicenter phase I trial of metronomic oral vinorelbine plus cisplatin in patients with NSCLC. Cancer Chemother Pharmacol 2011, 67:12391245. | Article I PubMed

64. Gorn M, Habermann CR, Anige M, Thom I, Schuch G, Andritzky B, Brandl S, Burkholder I, Edler L, Hossfeld DK, Bokemeyer C, Laack E: A pilot study of docetaxel and trofosfamide as second-line ,metronomic' chemotherapy in the treatment of metastatic non-small cell lung cancer (NSCLC). Onkologie 2008, 31:185-189. | Article | PubMed

65. Correale P, Cerretani D, Remondo C, Martellucci I, Marsili S, La Placa M, Sciandivasci A, Paolelli L, Pascucci A, Rossi M, Di Bisceglie M, Giorgi G, Gotti G, Francini G: A novel metronomic chemotherapy regimen of weekly platinum and daily oral etoposide in high-risk non-small cell lung cancer patients. Oncol Rep 2006, 16:133-140. | Article I PubMed

66. Samaritani R, Corrado G, Vizza E, Sbiroli C: Cyclophosphamide "metronomic" chemotherapy for palliative treatment of a young patient with advanced epithelial ovarian cancer. BMC Cancer 2007, 7:65. | Article | PubMed Abstract | PubMed Full Text

67. Katsumata N, Yasuda M, Takahashi F, Isonishi S, Jobo T, Aoki D, Tsuda H, Sugiyama T, Kodama S, Kimura E, Ochiai K, Noda K: Dose-dense paclitaxel once a week in combination with carboplatin every 3 weeks for advanced ovarian cancer: a phase 3, open-label, randomised controlled trial. Lancet 2009, 374:1331-1338. | Article I PubMed

68. Osborne RJ, Filiaci V, Schink JC, Mannel RS, Alvarez Secord A, Kelley JL, Provencher D, Scott Miller D, Covens AL, Lage JM: Phase III trial of weekly methotrexate or pulsed dactinomycin for low-risk gestational trophoblastic neoplasia: a gynecologic oncology group study. J Clin Oncol 2011, 29:825-831. | Article | PubMed Abstract | PubMed Full Text

69. Suvannasankha A, Fausel C, Juliar BE, Yiannoutsos CT, Fisher WB, Ansari RH, Wood LL, Smith GG, Cripe LD, Abonour R: Final report of toxicity and efficacy of a phase II study of oral cyclophosphamide, thalidomide, and prednisone for patients with relapsed or refractory multiple myeloma: A Hoosier Oncology Group Trial, HEM0121. Oncologist 2007, 12:99-106. | Article I PubMed

70. Greiner J, Kufer R, Reske SN, Martin V, Dohner H, Ringhoffer M: Metronomic treatment with low-dose trofosfamide leads to a longterm remission in a patient with docetaxel-refractory advanced metastatic prostate cancer. Case Report Med 2010, 2010:395720. | Article | PubMed Abstract | PubMed Full Text

71. Nicolini A, Mancini P, Ferrari P, Anselmi L, Tartarelli G, Bonazzi V, Carpi A, Giardino R: Oral low-dose cyclophosphamide in metastatic hormone refractory prostate cancer (MHRPC). Biomed Pharmacother 2004, 58:447-450. | Article | PubMed

72. Lord R, Nair S, Schache A, Spicer J, Somaihah N, Khoo V, Pandha H: Low dose metronomic oral cyclophosphamide for hormone resistant prostate cancer: a phase II study. J Urol 2007, 177:2136-2140; discussion 2140. | Article I PubMed

73. Nelius T, Klatte T, de Riese W, Haynes A, Filleur S: Clinical outcome of patients with docetaxel-resistant hormone-refractory prostate cancer treated with second-line cyclophosphamide-based 
metronomic chemotherapy. Med Oncol 2010, 27:363-367. | Article I PubMed

74. Fontana A, Galli L, Fioravanti A, Orlandi P, Galli C, Landi L, Bursi S, Allegrini G, Fontana E, Di Marsico R, Antonuzzo A, D'Arcangelo M, Danesi R, Del Tacca M, Falcone A, Bocci G: Clinical and pharmacodynamic evaluation of metronomic cyclophosphamide, celecoxib, and dexamethasone in advanced hormone-refractory prostate cancer. Clin Cancer Res 2009, 15:4954-4962. | Article | PubMed

75. Facchini G, Caraglia M, Morabito A, Marra M, Piccirillo MC, Bochicchio AM, Striano S, Marra L, Nasti G, Ferrari E, Leopardo D, Vitale G, Gentilini D, Tortoriello A, Catalano A, Budillon A, Perrone F, laffaioli RV: Metronomic administration of zoledronic acid and taxotere combination in castration resistant prostate cancer patients: phase I ZANTE trial. Cancer Biol Ther 2010, 10:543-548. | Article | PubMed

76. Glode LM, Barqawi A, Crighton F, Crawford ED, Kerbel R: Metronomic therapy with cyclophosphamide and dexamethasone for prostate carcinoma. Cancer 2003, 98:1643-1648. | Article | PubMed

77. Gebbia V, Serretta V, Borsellino N, Valerio MR: Salvage therapy with oral metronomic cyclophosphamide and methotrexate for castration-refractory metastatic adenocarcinoma of the prostate resistant to docetaxel. Urology 2011, 78:1125-1130. | Article | PubMed

78. Walter B, Schrettenbrunner I, Vogelhuber M, Grassinger J, Bross K, Wilke J, Suedhoff T, Berand A, Wieland WF, Rogenhofer S, Andreesen $R$, Reichle A: Pioglitazone, etoricoxib, interferon-alpha, and metronomic capecitabine for metastatic renal cell carcinoma: final results of a prospective phase II trial. Med Oncol 2012, 29:799-805. | Article | PubMed

79. Vogt T, Hafner C, Bross K, Bataille F, Jauch KW, Berand A, Landthaler $M$, Andreesen R, Reichle A: Antiangiogenetic therapy with pioglitazone, rofecoxib, and metronomic trofosfamide in patients with advanced malignant vascular tumors. Cancer 2003, 98:2251-2256. | Article | PubMed

80. Coras B, Hafner C, Reichle A, Hohenleutner U, Szeimies RM, Landthaler M, Vogt T: Antiangiogenic therapy with pioglitazone, rofecoxib, and trofosfamide in a patient with endemic kaposi sarcoma. Arch Dermatol 2004, 140:1504-1507. | Article | PubMed

81. Italiano A, Toulmonde M, Lortal B, Stoeckle E, Garbay D, Kantor $G$, Kind M, Coindre JM, Bui B: „Metronomic“ chemotherapy in advanced soft tissue sarcomas. Cancer Chemother Pharmacol 2010, 66:197-202. | Article | PubMed

82. Reichle A, Bross K, Vogt T, Bataille F, Wild P, Berand A, Krause SW, Andreesen R: Pioglitazone and rofecoxib combined with angiostatically scheduled trofosfamide in the treatment of far-advanced melanoma and soft tissue sarcoma. Cancer 2004, 101:2247-2256. | Article | PubMed

83. Ross $P$, Nicolson $M$, Cunningham $D$, Valle J, Seymour $M$, Harper P, Price T, Anderson $\mathrm{H}$, Iveson T, Hickish T, Lofts F, Norman A: Prospective randomized trial comparing mitomycin, cisplatin, and protracted venous-infusion fluorouracil (PVI 5-FU) with epirubicin, cisplatin, and PVI 5-FU in advanced esophagogastric cancer. J Clin Oncol 2002, 20:1996-2004. | Article | PubMed

84. Jurado JM, Sanchez A, Pajares B, Perez E, Alonso L, Alba E: Combined oral cyclophosphamide and bevacizumab in heavily pre-treated ovarian cancer. Clin Trans/ Oncol 2008, 10:583-586. | Article | PubMed

85. Aigner J, Bischofs E, Hallscheidt P, Sohn C, Schneeweiss A, Eichbaum $M$ : Long-term remission in a patient with heavily pretreated, advanced ovarian cancer achieved by bevacizumab and metronomic cyclophosphamide treatment. Anticancer Drugs 2011, 22:10301033. | Article | PubMed

86. Garcia AA, Hirte H, Fleming G, Yang D, Tsao-Wei DD, Roman L, Groshen S, Swenson S, Markland F, Gandara D, Scudder S, Morgan R, Chen $\mathrm{H}$, Lenz HJ, Oza AM: Phase II clinical trial of bevacizumab and low-dose metronomic oral cyclophosphamide in recurrent ovarian cancer: a trial of the California, Chicago, and Princess Margaret Hospital phase II consortia. J Clin Oncol 2008, 26:76-82. | Article | PubMed
87. Garcia-Saenz JA, Martin M, Calles A, Bueno C, Rodriguez L, Bobokova J, Custodio A, Casado A, Diaz-Rubio E: Bevacizumab in combination with metronomic chemotherapy in patients with anthracyclineand taxane-refractory breast cancer. J Chemother 2008, 20:632639. | Article | PubMed

88. Dellapasqua S, Bertolini F, Bagnardi V, Campagnoli E, Scarano E, Torrisi R, Shaked Y, Mancuso P, Goldhirsch A, Rocca A, Pietri E, Colleoni $\mathrm{M}$ : Metronomic cyclophosphamide and capecitabine combined with bevacizumab in advanced breast cancer. J Clin Oncol 2008, 26:4899-4905. | Article | PubMed

89. Reardon DA, Desjardins A, Vredenburgh JJ, Gururangan S, Sampson $J \mathrm{H}$, Sathornsumetee S, McLendon RE, Herndon JE, 2nd, Marcello $\mathrm{JE}$, Norfleet J, Friedman AH, Bigner DD, Friedman HS: Metronomic chemotherapy with daily, oral etoposide plus bevacizumab for recurrent malignant glioma: a phase II study. Br J Cancer 2009, 101:1986-1994. | Article | PubMed Abstract | PubMed Full Text

90. Treiber G, Wex T, Malfertheiner P: Impact of different anticancer regimens on biomarkers of angiogenesis in patients with advanced hepatocellular cancer. J Cancer Res Clin Oncol 2009, 135:271-281. | Article I PubMed

91. Montagna E, Cancello G, Torrisi R, Rizzo S, Scarano E, Colleoni M: Lapatinib and metronomic capecitabine combination in an HER2positive inflammatory breast cancer patient: a case report. Ann Oncol 2010, 21:667-668. | Article | PubMed

92. Ruan J, Martin P, Coleman M, Furman RR, Cheung K, Faye A, Elstrom $\mathrm{R}$, Lachs M, Hajjar KA, Leonard JP: Durable responses with the metronomic rituximab and thalidomide plus prednisone, etoposide, procarbazine, and cyclophosphamide regimen in elderly patients with recurrent mantle cell lymphoma. Cancer 2010, 116:26552664. | Article | PubMed Abstract | PubMed Full Text

93. Bellmunt J, Trigo JM, Calvo E, Carles J, Perez-Gracia JL, Rubio J, Virizuela JA, Lopez R, Lazaro M, Albanell J: Activity of a multitargeted chemo-switch regimen (sorafenib, gemcitabine, and metronomic capecitabine) in metastatic renal-cell carcinoma: a phase $\mathbf{2}$ study (SOGUG-02-06). Lancet Oncol 2010, 11:350-357. | Article | PubMed

94. Hsu CH, Shen YC, Lin ZZ, Chen PJ, Shao YY, Ding YH, Hsu C, Cheng AL: Phase II study of combining sorafenib with metronomic tegafur/ uracil for advanced hepatocellular carcinoma. J Hepatol 2010, 53:126-131. | Article | PubMed

95. Orlando L, Cardillo A, Ghisini R, Rocca A, Balduzzi A, Torrisi R, Peruzzotti G, Goldhirsch A, Pietri E, Colleoni M: Trastuzumab in combination with metronomic cyclophosphamide and methotrexate in patients with HER-2 positive metastatic breast cancer. BMC Cancer 2006, 6:225. | Article | PubMed Abstract | PubMed Full Text

96. Ogata $\mathrm{Y}$, Mori S, Ishibashi N, Akagi $\mathrm{Y}$, Ushijima M, Murakami $\mathrm{H}$, Fukushima T, Shirouzu K: Metronomic chemotherapy using weekly low-dosage CPT-11 and UFT as postoperative adjuvant therapy in colorectal cancer at high risk to recurrence. J Exp Clin Cancer Res 2007, 26:475-482. | Pdf | PubMed

97. Clarke JL, Iwamoto FM, Sul J, Panageas K, Lassman AB, DeAngelis LM, Hormigo A, Nolan CP, Gavrilovic I, Karimi S, Abrey LE: Randomized phase II trial of chemoradiotherapy followed by either dose-dense or metronomic temozolomide for newly diagnosed glioblastoma. J Clin Oncol 2009, 27:3861-3867. | Article | PubMed Abstract | PubMed Full Text

98. Lechleider RJ, Arlen PM, Tsang KY, Steinberg SM, Yokokawa J, Cereda V, Camphausen K, Schlom J, Dahut WL, Gulley JL: Safety and immunologic response of a viral vaccine to prostate-specific antigen in combination with radiation therapy when metronomic-dose interleukin 2 is used as an adjuvant. Clin Cancer Res 2008, 14:52845291. | Article | PubMed Abstract | PubMed Full Text

99. Ellis GK, Barlow WE, Gralow JR, Hortobagyi GN, Russell CA, Royce $M E$, Perez EA, Lew D, Livingston RB: Phase III comparison of standard doxorubicin and cyclophosphamide versus weekly doxorubicin and daily oral cyclophosphamide plus granulocyte colony-stimulating factor as neoadjuvant therapy for inflammatory and locally advanced breast cancer: SWOG 0012. J Clin Oncol 2011, 29:1014-1021. | Article | PubMed Abstract | PubMed Full Text 
100. Masferrer JL, Leahy KM, Koki AT, Zweifel BS, Settle SL, Woerner BM, Edwards DA, Flickinger AG, Moore RJ, Seibert K: Antiangiogenic and antitumor activities of cyclooxygenase-2 inhibitors. Cancer Res 2000, 60:1306-1311. | Article | PubMed

101. Zhu J, Song X, Lin HP, Young DC, Yan S, Marquez VE, Chen CS: Using cyclooxygenase- $\mathbf{2}$ inhibitors as molecular platforms to develop a new class of apoptosis-inducing agents. J Nat/ Cancer Inst 2002, 94:1745-1757. | Article | PubMed

102. Koki AT, Masferrer JL: Celecoxib: a specific COX-2 inhibitor with anticancer properties. Cancer Control 2002, 9:28-35. | Pdf | PubMed

103. van Wijngaarden J, van Beek E, van Rossum $G$, van der Bent $C$, Hoekman K, van der Pluijm G, van der Pol MA, Broxterman HJ, van Hinsbergh VW, Lowik CW: Celecoxib enhances doxorubicin-induced cytotoxicity in MDA-MB231 cells by NF-kappaB-mediated increase of intracellular doxorubicin accumulation. Eur J Cancer 2007, 43:433-442. | Article | PubMed

104. Bertagnolli MM, Eagle CJ, Zauber AG, Redston M, Solomon SD, Kim K, Tang J, Rosenstein RB, Wittes J, Corle D, Hess TM, Woloj GM, Boisserie F, Anderson WF, Viner JL, Bagheri D, Burn J, Chung DC, Dewar T, Foley TR, Hoffman N, Macrae F, Pruitt RE, Saltzman JR, Salzberg B, Sylwestrowicz T, Gordon GB, Hawk ET: Celecoxib for the prevention of sporadic colorectal adenomas. N Engl J Med 2006, 355:873-884. | Article | PubMed

105. Rothwell PM, Wilson M, Elwin CE, Norrving B, Algra A, Warlow CP, Meade TW: Long-term effect of aspirin on colorectal cancer incidence and mortality: 20-year follow-up of five randomised trials. Lancet 2010, 376:1741-1750. | Article | PubMed

106. Jiralerspong S, Palla SL, Giordano SH, Meric-Bernstam F, Liedtke C, Barnett CM, Hsu L, Hung MC, Hortobagyi GN, Gonzalez-Angulo AM: Metformin and pathologic complete responses to neoadjuvant chemotherapy in diabetic patients with breast cancer. J Clin Oncol 2009, 27:3297-3302. | Article | PubMed Abstract | PubMed Full Text

107. Gonzalez-Angulo AM, Meric-Bernstam F: Metformin: a therapeutic opportunity in breast cancer. Clin Cancer Res 2010, 16:1695-1700. | Article | PubMed Abstract | PubMed Full Text

108. Hirsch HA, lliopoulos D, Tsichlis PN, Struhl K: Metformin selectively targets cancer stem cells, and acts together with chemotherapy to block tumor growth and prolong remission. Cancer Res 2009, 69:7507-7511. | Article | PubMed Abstract | PubMed Full Text

109. Rozengurt E, Sinnett-Smith J, Kisfalvi K: Crosstalk between insulin/ insulin-like growth factor-1 receptors and $\mathrm{G}$ protein-coupled receptor signaling systems: a novel target for the antidiabetic drug metformin in pancreatic cancer. Clin Cancer Res 2010, 16:25052511. | Article | PubMed Abstract | PubMed Full Text

110. Jalving M, Gietema JA, Lefrandt JD, de Jong S, Reyners AK, Gans RO, de Vries EG: Metformin: taking away the candy for cancer? Eur J Cancer 2010, 46:2369-2380. | Article | PubMed

111. Niehues T, Horneff G, Megahed M, Schroten H, Wahn V: Complete regression of AIDS-related Kaposi's sarcoma in a child treated with highly active antiretroviral therapy. AIDS 1999, 13:1148-1149. | Article I PubMed

112. Gills JJ, Lopiccolo J, Tsurutani J, Shoemaker RH, Best CJ, Abu-Asab MS, Borojerdi J, Warfel NA, Gardner ER, Danish M, Hollander MC, Kawabata S, Tsokos M, Figg WD, Steeg PS, Dennis PA: Nelfinavir, A lead HIV protease inhibitor, is a broad-spectrum, anticancer agent that induces endoplasmic reticulum stress, autophagy, and apoptosis in vitro and in vivo. Clin Cancer Res 2007, 13:5183-5194. | Article | PubMed

113. Brunner TB, Geiger M, Grabenbauer GG, Lang-Welzenbach M, Mantoni TS, Cavallaro A, Sauer R, Hohenberger W, McKenna WG: Phase I trial of the human immunodeficiency virus protease inhibitor nelfinavir and chemoradiation for locally advanced pancreatic cancer. J Clin Oncol 2008, 26:2699-2706. | Article | PubMed

114. Gupta AK, Cerniglia GJ, Mick R, McKenna WG, Muschel RJ: HIV protease inhibitors block Akt signaling and radiosensitize tumor cells both in vitro and in vivo. Cancer Res 2005, 65:8256-8265. | Article | PubMed
115. Yki-Jarvinen H: Thiazolidinediones. N Engl J Med 2004, 351:11061118. | Article | PubMed

116. Panigrahy D, Singer S, Shen LQ, Butterfield CE, Freedman DA, Chen EJ, Moses MA, Kilroy S, Duensing S, Fletcher C, Fletcher JA, Hlatky L, Hahnfeldt P, Folkman J, Kaipainen A: PPARgamma ligands inhibit primary tumor growth and metastasis by inhibiting angiogenesis. J Clin Invest 2002, 110:923-932. | Article | PubMed Abstract | PubMed Full Text

117. Grabacka M, Plonka PM, Urbanska K, Reiss K: Peroxisome proliferator-activated receptor alpha activation decreases metastatic potential of melanoma cells in vitro via down-regulation of Akt. Clin Cancer Res 2006, 12:3028-3036. | Article | PubMed

118. Grommes C, Landreth GE, Heneka MT: Antineoplastic effects of peroxisome proliferator-activated receptor gamma agonists. Lancet Oncol 2004, 5:419-429. | Article | PubMed

119. Shim JS, Matsui Y, Bhat S, Nacev BA, Xu J, Bhang HE, Dhara S, Han $\mathrm{KC}$, Chong CR, Pomper MG, So A, Liu JO: Effect of nitroxoline on angiogenesis and growth of human bladder cancer. J Nat/ Cancer Inst 2010, 102:1855-1873. | Article | PubMed Abstract | PubMed Full Text

120. Shichiri M, Fukai N, Kono Y, Tanaka Y: Rifampicin as an oral angiogenesis inhibitor targeting hepatic cancers. Cancer Res 2009, 69:4760-4768. | Article | PubMed

121. Gasparini G, Morabito A, Magnani E, Gattuso D, Capaccetti B, Alberti AM: Thalidomide: an old sedative-hypnotic with anticancer activity? Curr Opin Investig Drugs 2001, 2:1302-1308. | Article | PubMed

122. Eisen T, Boshoff C, Mak I, Sapunar F, Vaughan MM, Pyle L, Johnston SR, Ahern R, Smith IE, Gore ME: Continuous low dose Thalidomide: a phase II study in advanced melanoma, renal cell, ovarian and breast cancer. Br J Cancer 2000, 82:812-817. | Article | PubMed Abstract | PubMed Full Text

123. Motzer RJ, Berg W, Ginsberg M, Russo P, Vuky J, Yu R, Bacik J, Mazumdar M: Phase II trial of thalidomide for patients with advanced renal cell carcinoma. J Clin Oncol 2002, 20:302-306. | Article | PubMed

124. Ezekowitz RA, Mulliken JB, Folkman J: Interferon alfa-2a therapy for life-threatening hemangiomas of infancy. N Engl J Med 1992, 326:1456-1463. | Article | PubMed

125. Gatenby RA: A change of strategy in the war on cancer. Nature 2009, 459:508-509. | Article | PubMed

126. Emmenegger U, Francia G, Shaked Y, Kerbel RS: Metronomic chemotherapy: principles and lessons learned from applications in the treatment of metastatic prostate cancer. Recent Results Cancer Res 2010, 180:165-183. | Article | PubMed

127. Pasquier E, Kavallaris M, Andre N: Metronomic chemotherapy: new rationale for new directions. Nat Rev Clin Oncol 2010, 7:455-465. I Article | PubMed

128. Doloff JC, Waxman DJ: VEGF receptor inhibitors block ability of metronomically dosed cyclophosphamide to activate innate immunity-induced tumor regression. Cancer Res 2012, 72: 103-1115. | Article I PubMed

129. Sadeghi N, Abbruzzese JL, Yeung SCJ, Hassan M, Li D: Metformin use is associated with better survival of diabetic patients with pancreatic cancer. Clin Cancer Res 2012, 18:2905-2912. | Article | PubMed

\section{Citation:}

Mross K and Steinbild S: Metronomic anticancer therapy - an ongoing treatment option for advanced cancer patients. journal of Cancer Therapeutics and Research 2012, 1:32.

http://dx.doi.org/10.7243/2049-7962-1-32 